\title{
Fostering socio-emotional learning through early childhood intervention
}

\author{
Christina F. Mondi ${ }^{1 *} \mathbb{D}$, Alison Giovanelli ${ }^{2}$ and Arthur J. Reynolds ${ }^{3}$
}

\section{*Correspondence:}

christina.mondi-

rago@childrens.harvard.edu;

mondi004@umn.edu

${ }^{1}$ Division of Developmental

Medicine, Brazelton

Touchpoints Center,

Boston Children's Hospital,

Harvard Medical School,

1295 Boylston St., Suite 320,

Boston, MA 02115, USA

Full list of author information

is available at the end of the article

\begin{abstract}
Educators and researchers are increasingly interested in evaluating and promoting socio-emotional learning (SEL) beginning in early childhood (Newman \& Dusunbury in 2015; Zigler \& Trickett in American Psychologist 33(9):789-798 https://doi.org/10.1037/ 0003-066X.33.9.789, 1978). Decades of research have linked participation in high-quality early childhood education (ECE) programs (e.g., public prekindergarten, Head Start) to multidimensional wellbeing. ECE programs also have demonstrated potential to be implemented at large scales with strong financial returns on investment. However, relatively few studies have investigated the effects of ECE programs on SEL, particularly compared to smaller-scale, skills-based SEL interventions. Furthermore, among studies that have examined SEL, there is a general lack of consensus about how to define and measure SEL in applied settings. The present paper begins to address these gaps in several ways. First, it discusses conceptual and methodological issues related to developmentally and culturally sensitive assessment of young children's socio-emotional functioning. Second, it reviews the empirical research literature on the impacts of three types of early childhood programs (general prekindergarten programs; multi-component prekindergarten programs; and universal skills-based interventions) on SEL. Finally, it highlights future directions for research and practice.
\end{abstract}

Keywords: Early childhood, Socio-emotional learning, Mental health, Prevention, Early intervention

\section{Fostering socio-emotional learning through early childhood programming}

What are the best ways to assess the effectiveness of early childhood intervention programs? This question has been debated for decades, and the answer has tremendous implications for public policy. During the mid-twentieth century, many research studies primarily examined whether intervention participation led to improvements in children's IQ scores. Some researchers, however, argued for a more multidimensional approach to assessing intervention outcomes. Edward Zigler, one of the architects of Head Start, notably proposed that the primary outcome of interest in early childhood interventions should be children's "social competence" (Raver \& Zigler, 1997; Zigler \& Trickett, 1978). Interest in social competence grew in the second half of the twentieth century, with numerous studies indicating that socio-emotional and motivational variables exert significant impacts on wellbeing in childhood and beyond (Greenberg et al.,

(c) The Author(s) 2021. This article is licensed under a Creative Commons Attribution 4.0 International License, which permits use, sharing, adaptation, distribution and reproduction in any medium or format, as long as you give appropriate credit to the original author(s) and the source, provide a link to the Creative Commons licence, and indicate if changes were made. The images or other third party material in this article are included in the article's Creative Commons licence, unless indicated otherwise in a credit line to the material. If material is not included in the article's Creative Commons licence and your intended use is not permitted by statutory regulation or exceeds the permitted use, you will need to obtain permission directly from the copyright holder. To view a copy of this licence, visit http://creativeco mmons.org/licenses/by/4.0/. 
2003; Jones et al., 2015). By the turn of the twenty-first century, a national sample of teachers reported that they believed that the ability to regulate emotions and behaviors is the most important component of school readiness (Rimm-Kaufman et al., 2000).

Today, educators and researchers continue to be interested in evaluating and promoting socio-emotional learning (SEL) starting in early childhood. Early childhood SEL skills develop rapidly, are uniquely malleable, and are strongly associated with later social, academic, cognitive, and health outcomes (Zins et al., 2007). Skills-based interventions that specifically target children's SEL have been a major area of investigation (McClelland et al., 2017). However, relatively less is known about the impacts of largescale early childhood education (ECE) programs on SEL, despite the potential of such programs to effect broad impacts. Furthermore, despite the growing enthusiasm surrounding the concept of SEL, many of the same methodological issues that Zigler and colleagues described in the 1970s still persist. Review of the literature reveals a lack of consensus among researchers and practitioners regarding how to define, evaluate, and promote SEL.

McCabe and Altamura (2011) previously reviewed the impact of a variety of preschool interventions on SEL, including both skills-based interventions and comprehensive classroom- and home-based programs. The authors reported that many programs were associated with short-term SEL benefits, but that there was a need for additional longitudinal research in this area. Notably, the authors did not explicate their review methodology or inclusion criteria, making it difficult to ascertain the representativeness and comprehensiveness of their findings. This limitation, combined with the publication of a number of studies since 2011, signal the need for an updated review of different intervention strategies for preschool-aged children.

The present paper reviews the most methodologically rigorous research that is available on the relationship between preschool intervention and SEL. We begin by discussing what the construct of SEL is (and is not) - a topic that that has been the subject of some debate and confusion in the literature. Having outlined a conceptual and methodological framework for SEL, we will then describe specific study aims and methods.

\section{Socio-emotional competencies in early childhood}

During the early 1990s, the Collaborative for Academic, Social, and Emotional Learning (CASEL) attempted to organize decades of empirical research on socio-emotional development into a socio-emotional learning (SEL) framework (Newman \& Dusunbury, 2015). Since then, the CASEL framework has been widely used by researchers, practitioners, and policymakers alike, informing the development of federal and state state legislation and learning standards.

According to CASEL researchers, SEL is the process of learning to "integrate thinking, feeling, and behaving to achieve important life tasks" (Zins et al., 2007, p. 194). SEL encompasses children's emerging abilities to "form close and secure... relationships; experience, regulate, and express emotions in socially and culturally appropriate ways; and explore the environment and learn-all in the context of family, community, and culture" (Yates et al., 2008, p. 2). CASEL's SEL framework is grounded in research on typical and atypical socio-emotional development and highlights five core competency areas: (a) self-awareness; (b) self-management; (c) social 
awareness; (d) relationship skills; and (e) responsible decision-making (Collaborative for Social \& Emotional Learning [CASEL], 2012; "Core SEL Competencies", 2019; Weissberg et al., 2016). These competences are outlined in Table 1.

Importantly, while CASEL's five core competency areas are common across most cultures, specific aspects of adaptive SEL functioning may vary based on race/ethnicity, language, socioeconomic status, and other cultural factors. Cognitive, linguistic, and behavioral traits that are considered to be adaptive and desirable in non-majority culture communities may be perceived as problematic or even pathological by majority culture educators (Phillips, 1993; West-Olatunji et al., 2008). These perceptions may be partially attributed to educators' own biases, and to disparities between the culture and structure of children's home and school environments (Boykin, 1983; Han \& Thomas, 2010; Ladson-Billings, 1995; McCarthy et al., 2006; Webb-Johnson, 2002). Thus, when assessing children's SEL, researchers and educators should carefully consider the role that culture plays in shaping children's behavior, and avoid conflating cultural behavioral differences with disorder.

\section{Conceptual and measurement issues}

\section{Distinguishing SEL from executive function}

SEL skills have often been referred to as "non-cognitive" skills in research and practice. Yet many researchers have argued that this designation is a misnomer, given that SEL skills are often grounded in skills related to cognition, learning, and memory. Among the most significant contributors to SEL are executive functioning (EF) skills, which include the cognitive processes necessary for planning, organizing, and problem-solving. Several studies have linked EF deficits to concurrent SEL deficits, and longitudinal work has indicated that EF skills in early childhood predict SEL competence later in life (e.g., Riggs et al., 2006). Thus, EF and SEL competencies, (including self-management, as identified by CASEL's framework) can be conceptualized as distinct but related, and at times overlapping, constructs.

Table 1 SEL core competencies (CASEL 2012)

\begin{tabular}{lll}
\hline Competency & Description & Examples \\
\hline Self-awareness & $\begin{array}{c}\text { The ability to accurately evaluate one's } \\
\text { own thoughts, emotions, abilities, } \\
\text { and values } \\
\text { The ability to effectively regulate one's } \\
\text { own thoughts, emotions, and actions } \\
\text { in different scenarios }\end{array}$ & $\begin{array}{l}\text { Emotion identification, self-confidence } \\
\text { Self-management }\end{array}$ \\
$\begin{array}{c}\text { The ability to empathize with individu- } \\
\text { als from diverse backgrounds and } \\
\text { to understand societal norms for } \\
\text { behavior }\end{array}$ & $\begin{array}{c}\text { Perspective-taking, showing respect } \\
\text { to others }\end{array}$ \\
& $\begin{array}{c}\text { The ability to initiate and maintain } \\
\text { healthy relationships }\end{array}$ & $\begin{array}{c}\text { Communicating clearly, constructively } \\
\text { navigating conflict }\end{array}$ \\
Relationship skills & $\begin{array}{c}\text { The ability to make healthy, informed } \\
\text { choices }\end{array}$ & $\begin{array}{c}\text { Identifying problems, analyzing the } \\
\text { potential consequences of a decision }\end{array}$ \\
Responsible decision-making &
\end{tabular}




\section{Distinguishing SEL from psychopathology}

Psychologists increasingly agree that mental health is most accurately conceptualized on a continuum, ranging from clinically significant psychopathology to psychological wellbeing or flourishing (Keyes, 2002). Within this model, mental health or wellbeing is conceptualized not only as the absence of psychopathology symptoms, but as the presence of competencies that enable individuals to withstand adversity and to work towards positive outcomes. As Darling-Churchill and Lippman (2016, p. 3) stated: "Problems and strengths do not fall neatly on a single continuum, and the absence of problems does not guarantee the presence of competencies; thus, it is important to measure both." From this perspective, it is imperative that researchers and practitioners avoid conflating emergent SEL deficits with psychopathology (Halle \& Darling-Churchill, 2016).

Children exhibiting emergent SEL deficits may or may not have comorbid psychiatric disorders. Children with diagnosable psychopathology must exhibit symptoms that coalesce into specific patterns and that are associated with significant functional impairment. The latter group would likely benefit from clinical treatment. Meanwhile, many children do not currently meet diagnostic criteria for a disorder, but exhibit emergent deficits in SEL skills relative to same-age peers (Jones et al., 2002; Wille et al., 2008). A multitude of factors may contribute to lagged SEL, including early deprivation or trauma, inconsistent caregiving, and cultural differences in socio-emotional expression. Children with emergent SEL deficits would likely benefit from broader-based interventions that provide opportunities for them to interact with high-quality caregivers, establish peer relationships, and practice SEL skills in the environments that they are already in (e.g., early care and education settings).

Emergent SEL deficits are distinct from clinical disorder; however, it is important to acknowledge the demonstrated link between early SEL deficits and long-term risk for the development of psychopathology. This link reflects the phenomenon of heterotypic continuity, in which an early behavior predicts the subsequent emergence of a different behavior in the same individual (Rutter et al., 2006). The concept of developmental cascades has been invoked as a potential mechanism for heterotypic continuity; in this case, an individual's early SEL competencies interact with other individual and environmental factors (e.g., genetic, family, school) over time, influencing his or her risk of developing psychopathology (Burke et al., 2005). For example, a child who is lagging in SEL may have negative interactions with caregivers and peers and fall behind academically. These experiences may, in turn, increase the child's probability of academic, psychological, and other difficulties over time. Conversely, a child who exhibits developmentally appropriate SEL will likely experience more social and academic success, which can lay foundations for lifelong wellbeing.

\section{Other measurement issues}

As noted above, it is critical that researchers utilize measures that assess children's SEL skills (as distinguished from EF skills or psychopathology symptoms).

Several additional issues merit consideration when assessing SEL in early childhood (Committee on Developmental Outcomes, 2008; Darling-Churchill \& Lippman, 2016; Halle \& Darling-Churchill, 2016). Measurement should ideally occur across multiple time-points, as longitudinal assessment allows for stronger inference of causal 
relationships. Collecting repeated measurements over time will also allow researchers to observe trajectories of socio-emotional development over time. Finally, collecting data from multiple informants is considered ideal in order to gain more comprehensive, reliable pictures of children's functioning. Integrating reports from different informants, who may perceive children's behaviors differently or observe different behaviors in different settings (e.g., home versus school), can be challenging; however, several methodological solutions to this problem have been proposed (e.g., Offord et al., 1996).

\section{Present review}

The present paper reviews the current state of the literature on SEL interventions for preschool-aged children. This review makes several unique contributions. First, whereas previous reviews have primarily focused on skills-based SEL interventions, this review compares and contrasts the effects of three types of early childhood interventions on SEL: (a) general prekindergarten programs; (b) multi-component prekindergarten programs; and (c) skills-based interventions. This review specifically focuses on universal programs in each of the three categories (e.g., programs that are not specifically targeted to children with emergent SEL deficits or psychopathology). Second, whereas several previous reviews have examined the effects of early intervention on child psychopathology (e.g., internalizing, externalizing symptoms), the current review examines SEL outcomes, defined as children's acquisition of developmentally appropriate social and emotional skills. Finally, rather than reviewing the entire literature, this review focuses on the most methodologically rigorous (e.g., peer-reviewed, longitudinal) extant research. Given these combined foci, the present review offers a thorough, up-to-date overview of the effects of different types of early childhood interventions on young children's SEL.

Notably, while we believe that it is imperative to evaluate the strength of programs' evidence bases using specific uniform criteria, our review reveals variable methodology and construct validity across individual studies, making it challenging to assess program efficacy in a reliable or systematic way. As such, we emphasize that the purpose of this review is not to make statements about the efficacy of individual programs, but rather to describe programs that are most promising and to identify knowledge gaps for future research to investigate.

\section{Method}

Having reviewed key conceptual and methodological issues, we will now describe our review of universal interventions for preschool-aged children. We conducted searches in Web of Science, PsycInfo, Google Scholar, and PubMed. Numerous search terms were employed, including ones referencing socio-emotional skills (e.g., "socio-emotional", "emotion regulation", "non-cognitive", "prosocial"), early childhood and ECE programming (e.g., "preschool," "Head Start"), and commonly used SEL measures (e.g., "Behavior Assessment System", "Conners"). Backwards and forward searches were conducted on landmark and highly cited articles.

Studies had to meet six inclusion criteria to be included in the present review. The purpose of these criteria was to identify the most methodologically rigorous studies on modern universal interventions and SEL. (1) studies had to be published in English in 
peer-reviewed journals by December 31, 2020. (2) Only studies that investigated prekindergarten interventions implemented in 1990 or later were included. (3) Interventions had to be universal (e.g., not specifically targeted to children with baseline SEL deficits or psychopathology) and delivered by laypeople (e.g., not researchers). (4) Critically, given that the focus of the present paper is the relations between intervention and SEL included, studies had to measure one or more SEL skills as previously defined. Studies were not included if they solely measured psychopathology outcomes (e.g., externalizing or internalizing symptoms, problem behaviors) or EF outcomes. (5) Studies had to assess children's SEL skills at a minimum of two time-points, as skill development over the course of intervention can only be examined within longitudinal research designs. (6) Studies had to include a comparison or control group.

The first and second authors independently reviewed the titles and abstracts of identified studies to determine whether they met inclusion criteria. During this review process, both authors also determined which intervention category applied to each study. General public prekindergarten programs were defined as publicly funded programs administered by state and local agencies. Multi-component ECE programs were defined as programs which provide multiple academic, family and social support services (e.g., Head Start, the Child-Parent Center (CPC) Program, The High/Scope Perry Preschool Project), typically in center-based settings. Skills-based SEL interventions were defined as discrete interventions aimed at enhancing children's SEL via direct skills instruction for children and/or their ECE caregivers (e.g., Al's Pals, The Incredible Years). In cases of disagreement, both authors reviewed and discussed until consensus was reached. Overall, based on these criteria, the following studies are included in the present review: (a) one empirical study of a general public prekindergarten program; (b) three empirical studies of multi-component ECE interventions; (c) 23 empirical studies of skills-based SEL interventions; (d) three systematic reviews or meta-analyses of multi-component ECE interventions; and (e) five systematic reviews or meta-analyses of SEL skills-based interventions. See Tables 2, 3, 4, 5 and 6 for details on these studies, including sample characteristics.

\section{Results}

\section{General public prekindergarten and multi-component ECE programs (Tables 2, 3, 4)}

General public prekindergarten and multi-component ECE programs (e.g., Head Start, the CPC program) are comprehensive ECE interventions, and stand in contrast to skills-based interventions which primarily target SEL. Nonetheless, there are several important distinctions between general public prekindergarten programs and multicomponent ECE programs (e.g., Head Start, the CPC Program). There is often significant variability in general prekindergarten models and populations served, both across and within public school districts in the United States (Phillips et al., 2017). Meanwhile, multi-component ECE programs typically incorporate similar program elements and serve comparable populations across sites. Multi-component programs often operate in center-based settings, and typically provide a wider range of support services for children and families than general public prekindergarten programs.

Despite the differences between general public prekindergarten and multi-component ECE programs, we present our findings for both program types simultaneously below. 


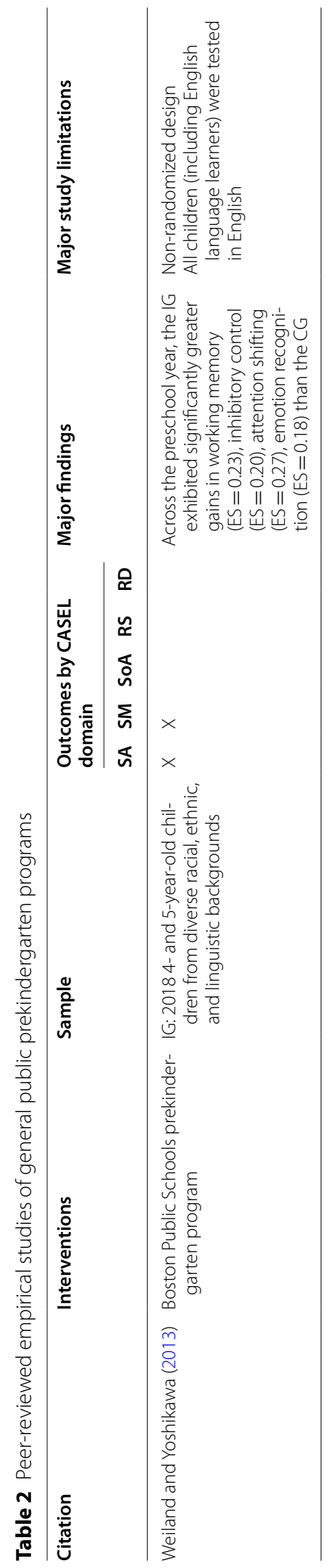




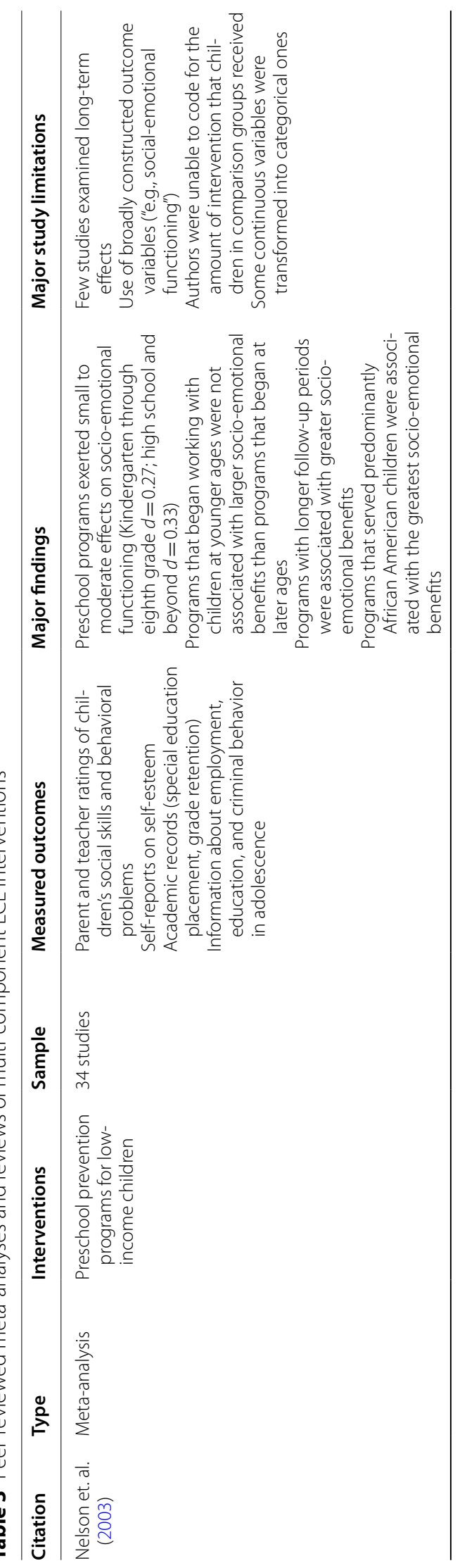




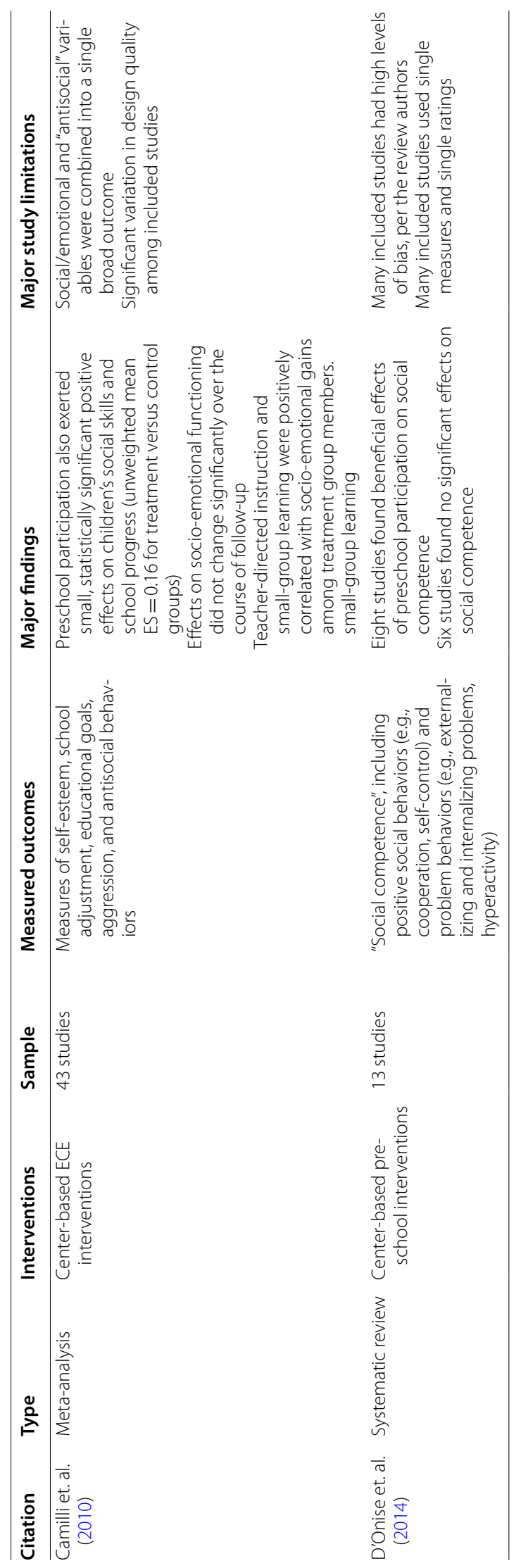




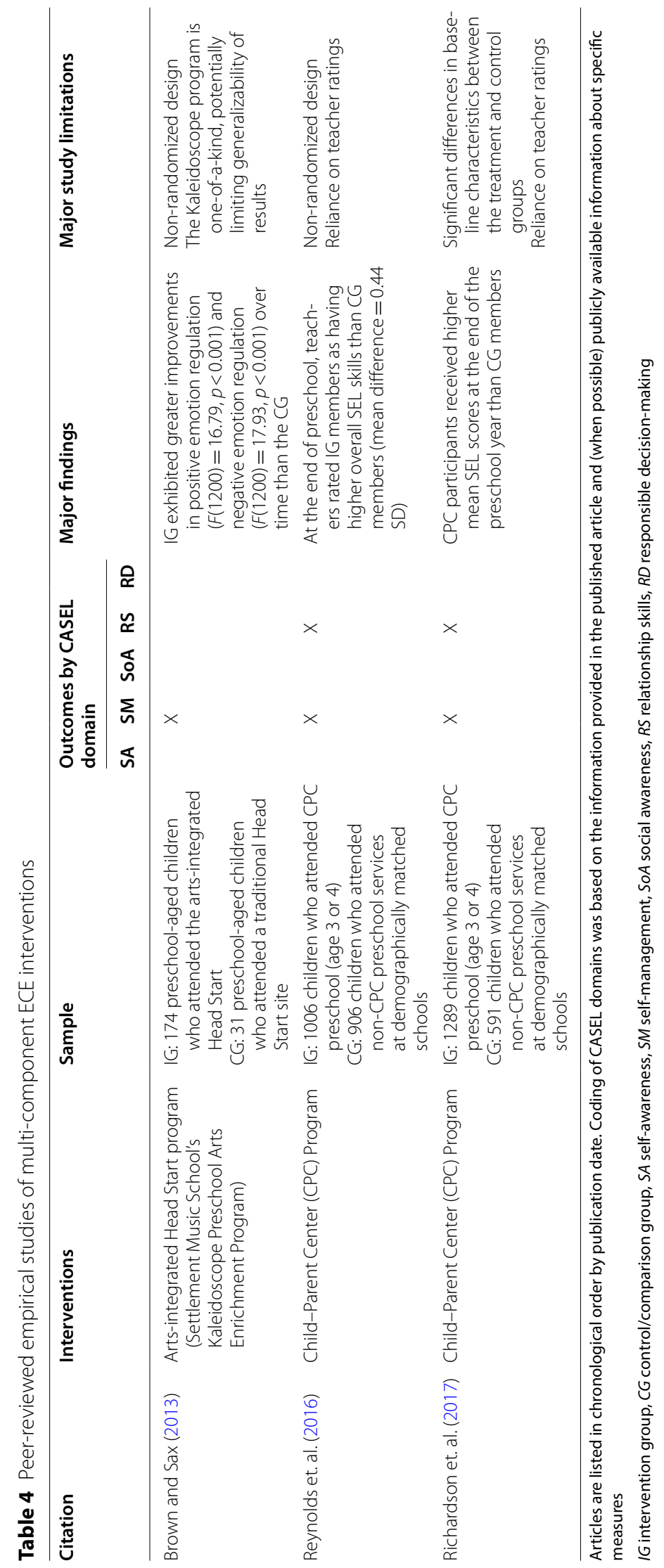




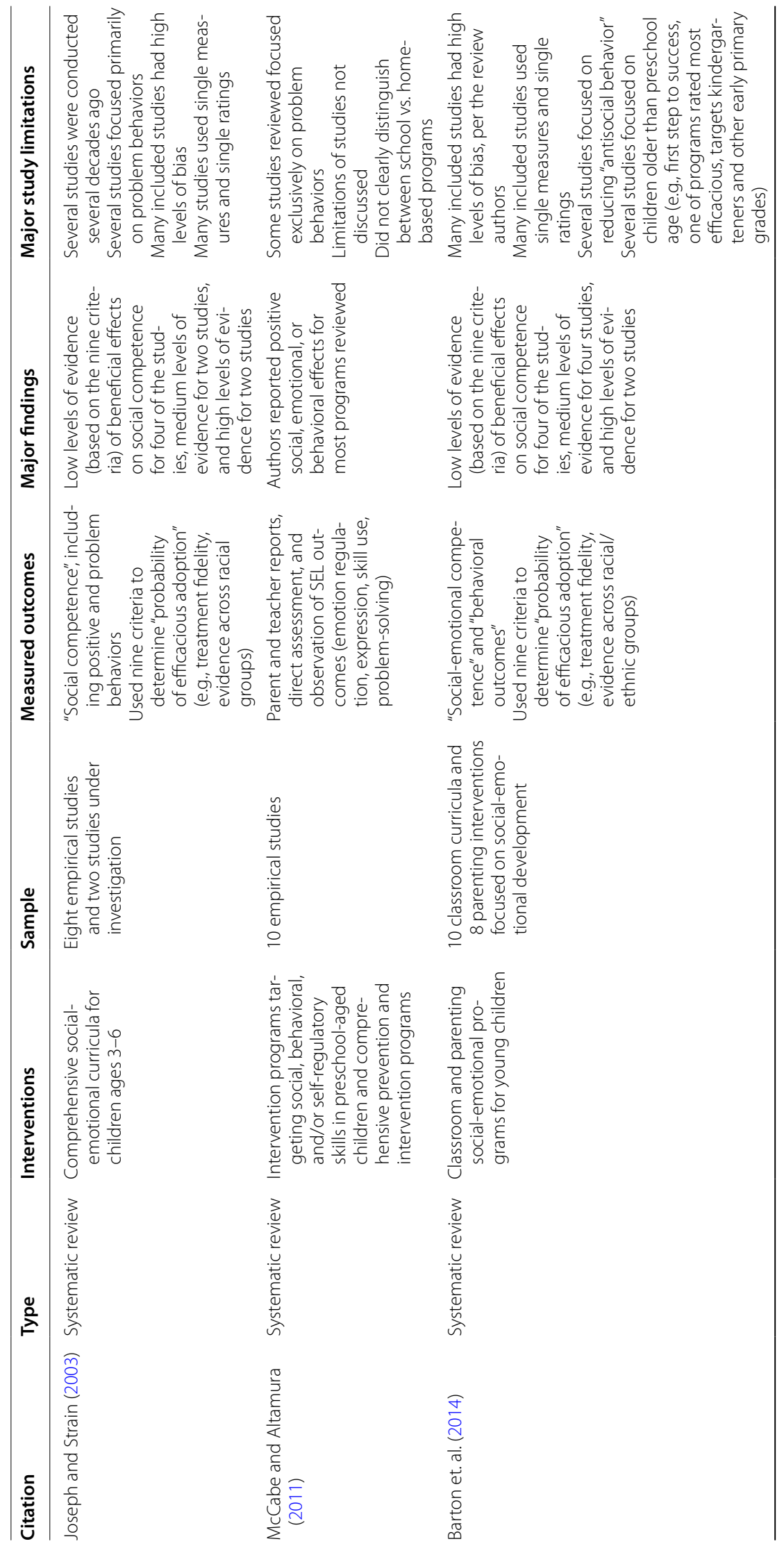




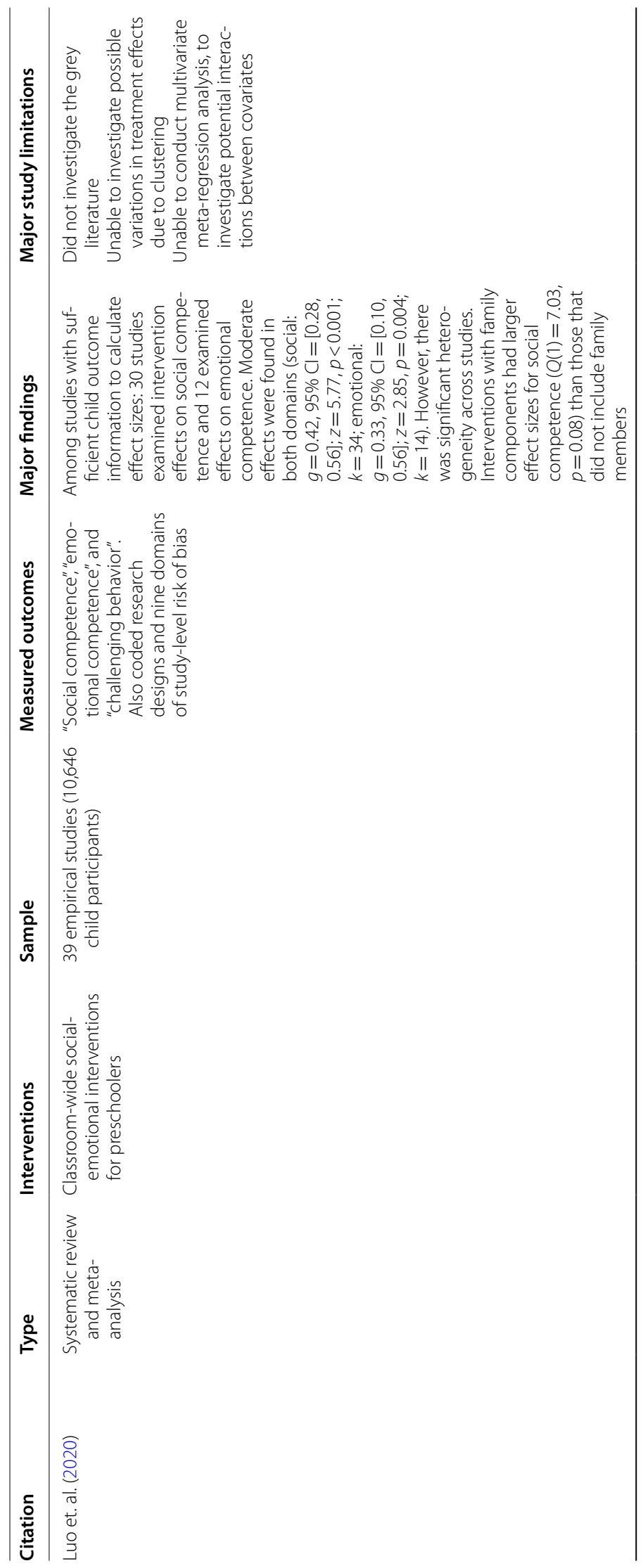




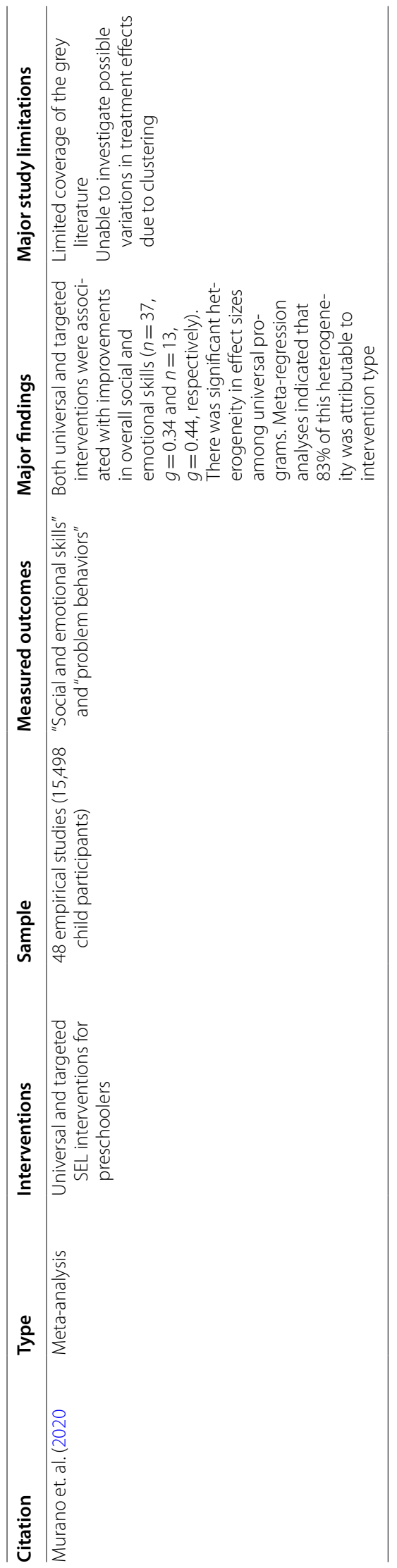




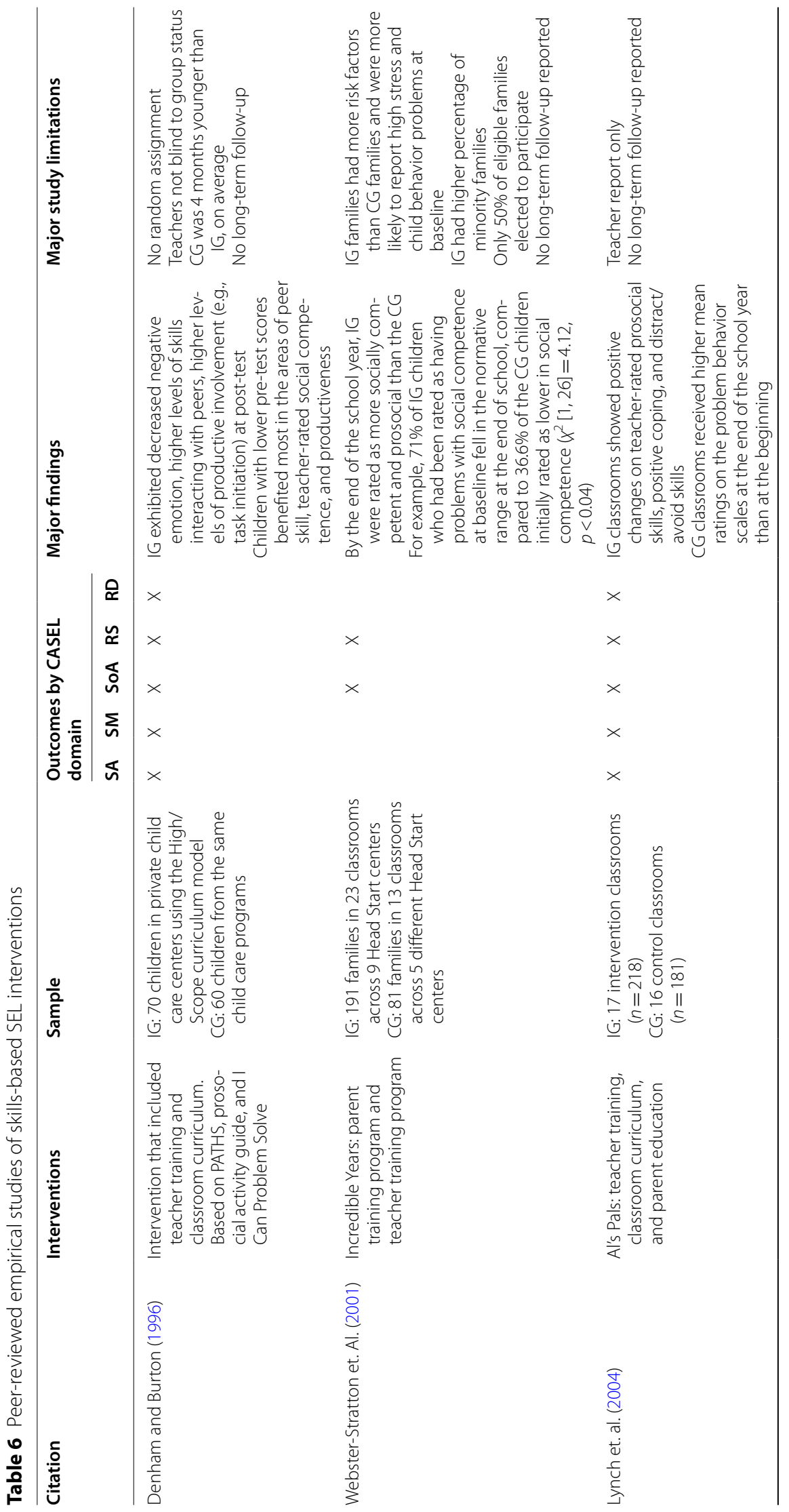




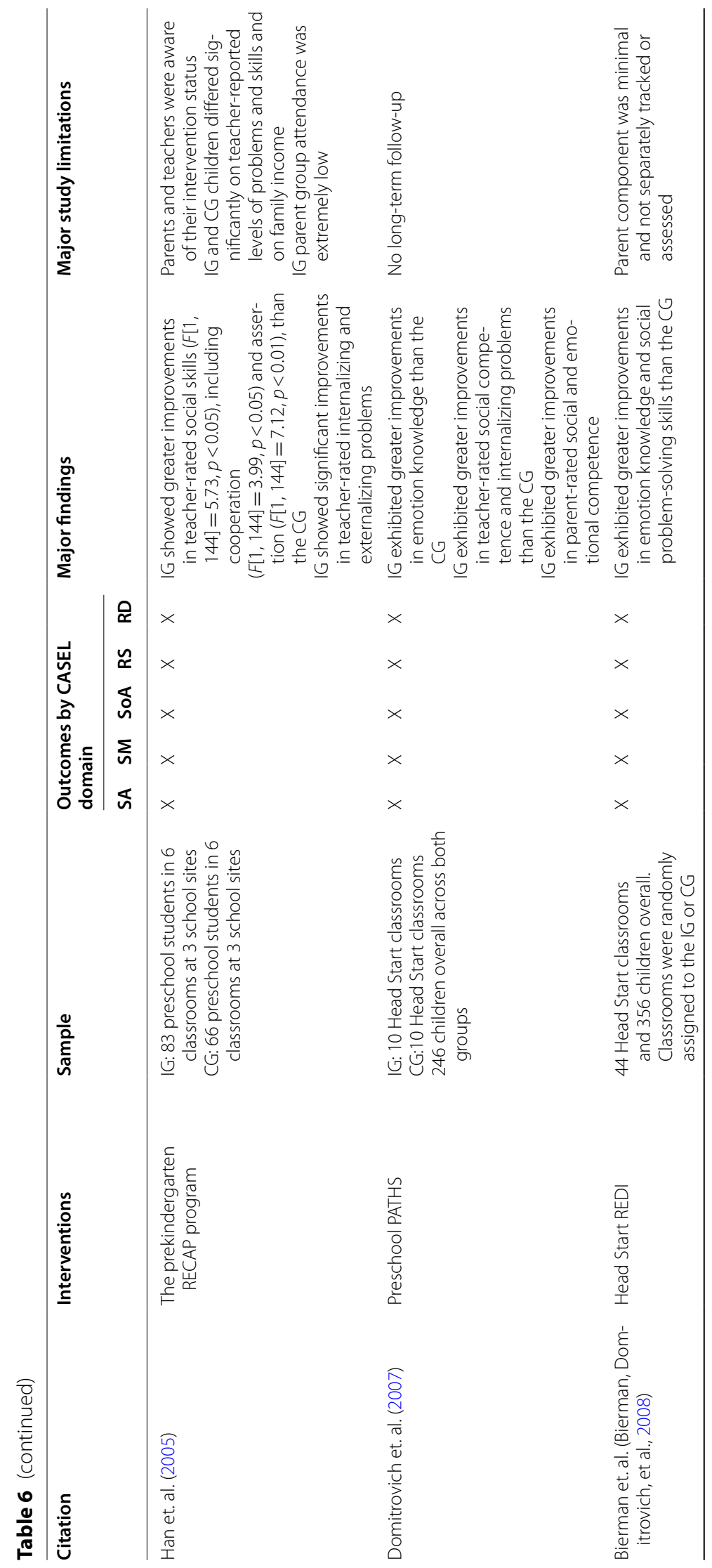




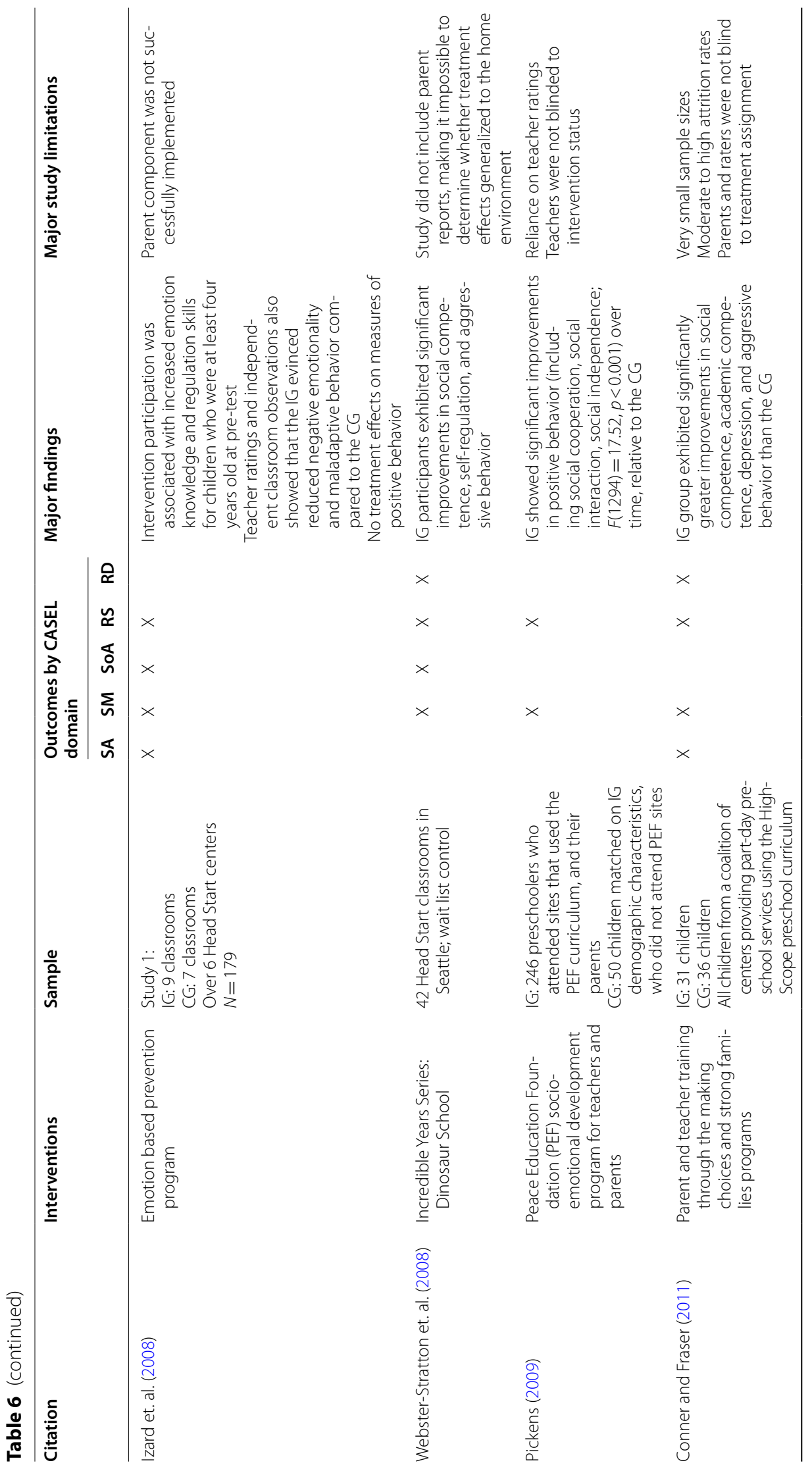




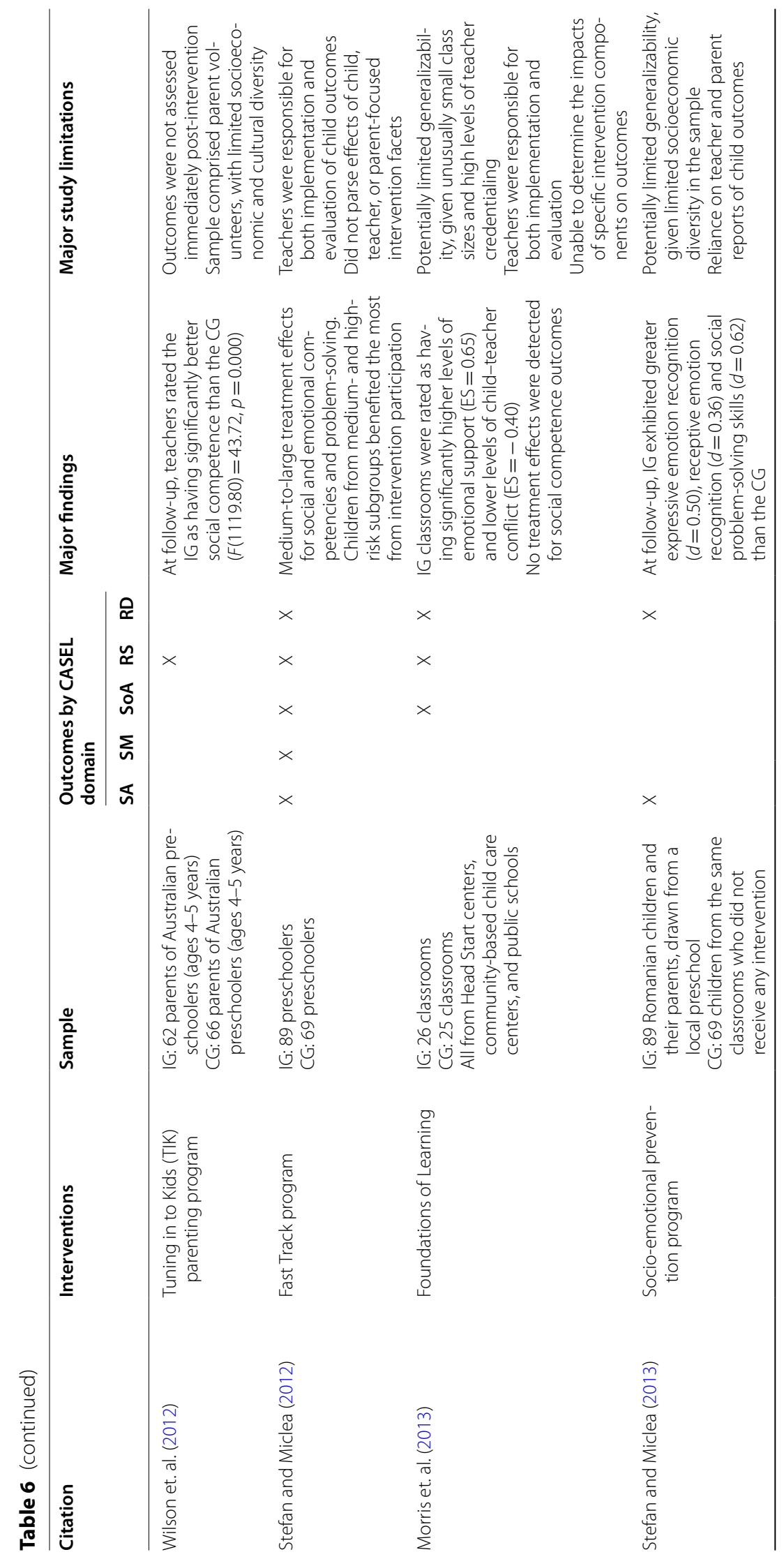




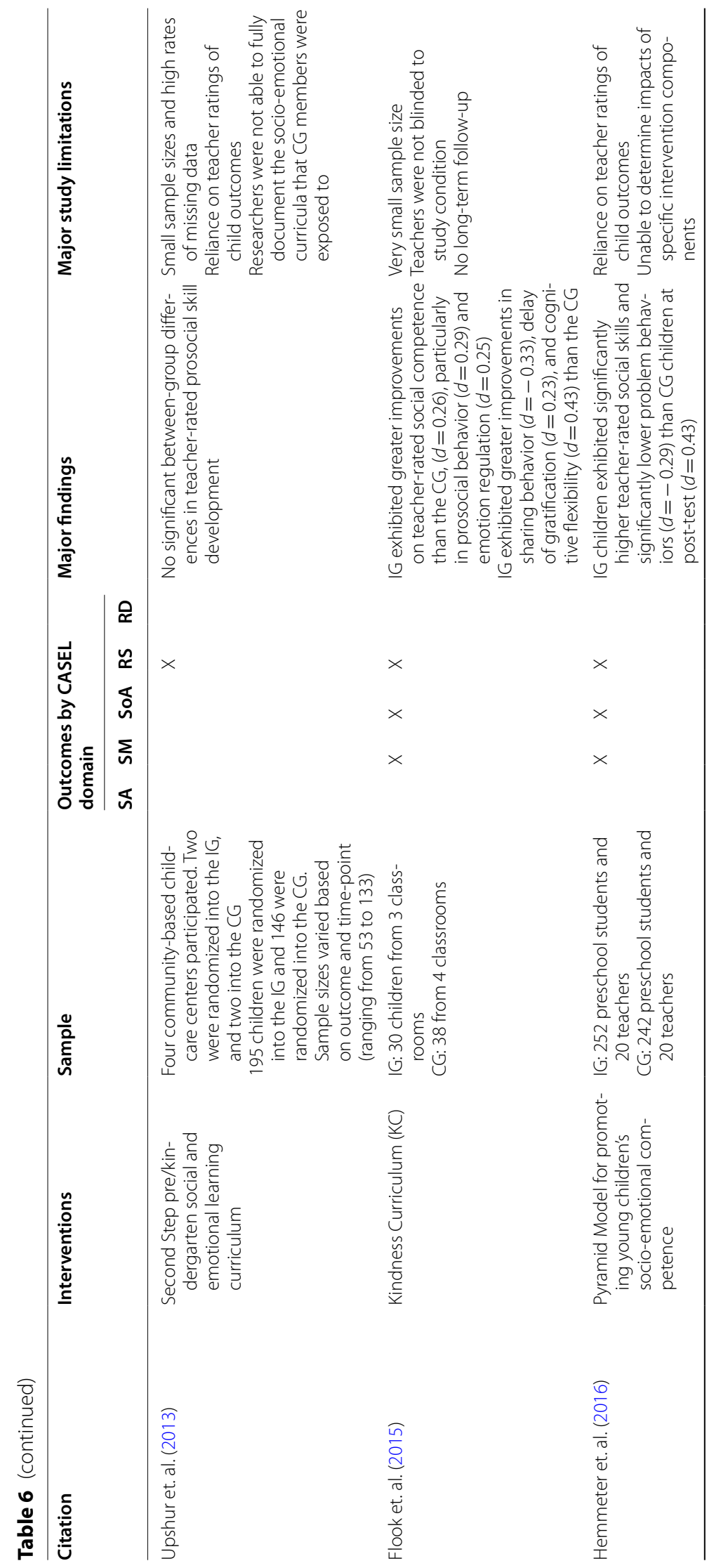




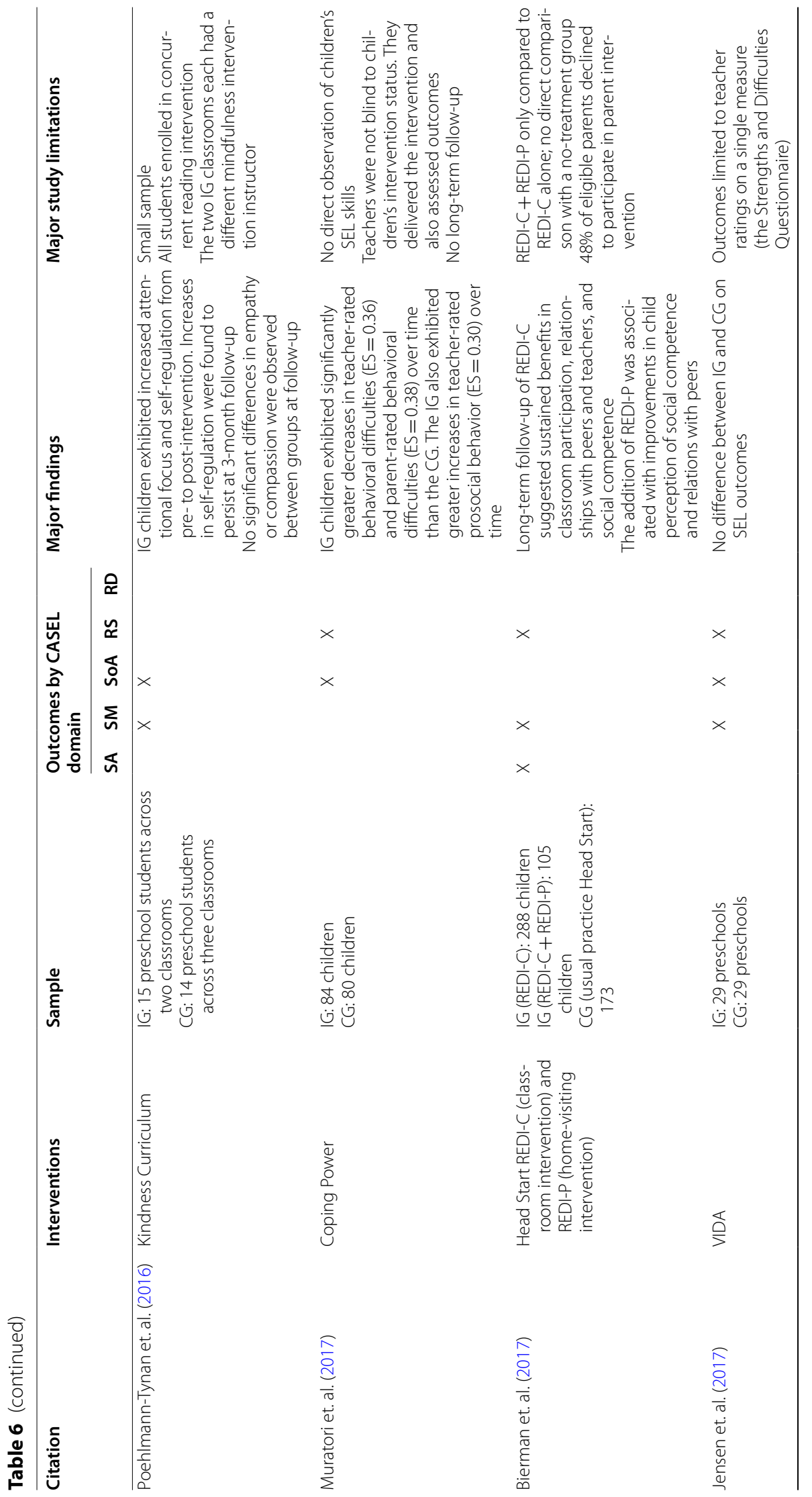




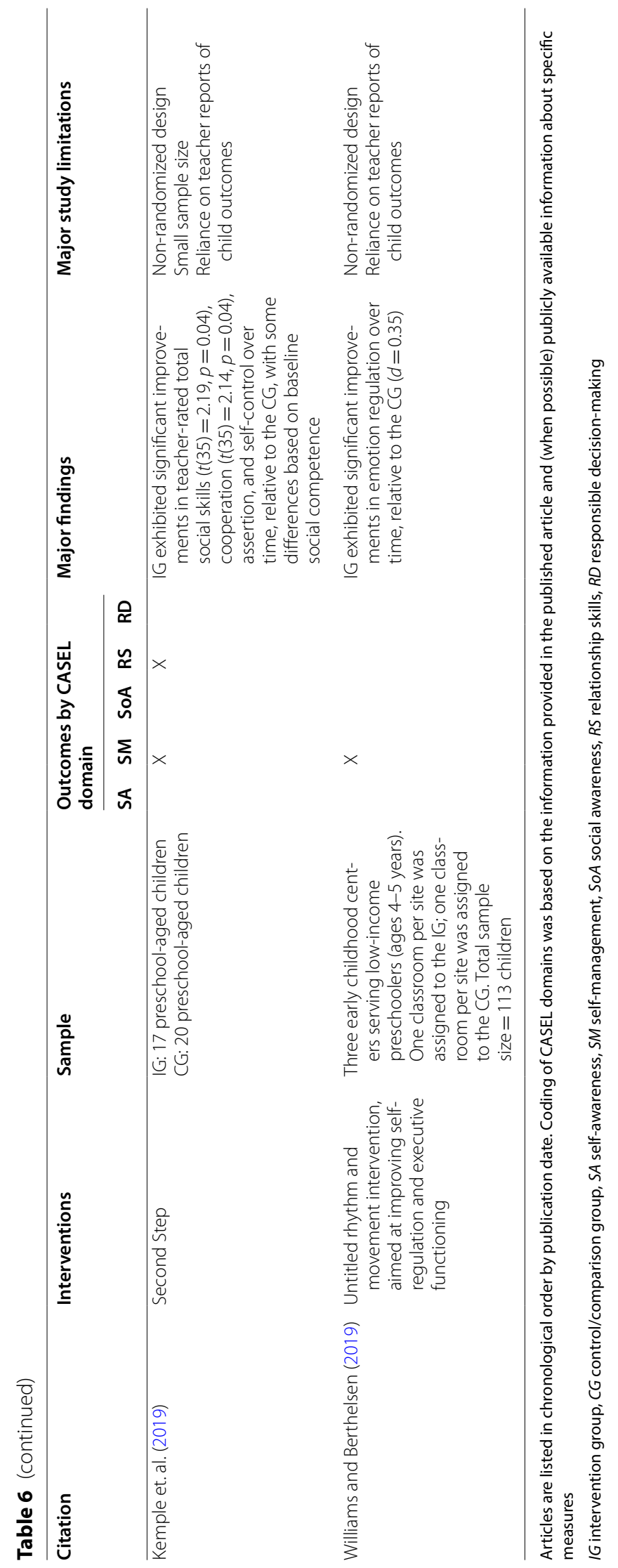


This is because, based on our review and to our knowledge, only one peer-reviewed study (Weiland \& Yoshikawa, 2013) has examined the effects of general public prekindergarten participation on SEL. A small number of studies have examined the relations between public prekindergarten participation and emotional and behavioral problems in childhood (e.g., internalizing and externalizing symptoms) (e.g., Gormley et al., 2011; Magnuson et al., 2007); however, as previously discussed, the focus of this review is on the relationship between intervention and SEL, not psychopathology symptoms. The lack of research on SEL in the context of public prekindergarten is a major gap that we will discuss in more depth later in this paper. In the interim, we present our findings on both types of non-SEL-skills-based interventions (general public prekindergarten and multi-component ECE programs).

\section{Meta-analyses and reviews (Table 3)}

Our review did not uncover any peer-reviewed meta-analyses or systematic reviews of the relations between public prekindergarten programming and SEL. On the contrary, several peer-reviewed meta-analyses and systematic reviews have investigated the effects of multi-component ECE programs on SEL. The authors of these publications have typically constructed outcome variables using a combination of measures assessing SEL skills, mental health symptoms, and outcomes from other domains that are related to socio-emotional functioning (e.g., special education placement, criminal justice system involvement). These publications will be briefly reviewed herein.

Nelson et. al. (2003) published one of the first meta-analyses examining preschool prevention programs for low-income children and families. Inclusion criteria included (1) presence of a prospective research design, (2) control or comparison group, and (3) at least one follow-up assessment in elementary school or beyond. In all, 34 qualifying interventions were identified. The authors reported that preschool programs exerted small to moderate effects on socio-emotional functioning in both the short-term (Kindergarten through eighth grade; $d=0.27$ ) and long-term (high school and beyond; $d=0.33$ ). Age at program entry was not related to program impacts; however, higher program dosage was linked to stronger effects on socio-emotional functioning. Results also indicated that African American children were more likely to participate in the most intensive interventions, and that programs that predominately served the latter group were associated with the greatest socio-emotional benefits.

Several years later, Camilli et. al. (2010) conducted another meta-analysis examining the effects of ECE participation on child outcomes. To be included in the meta-analysis, interventions had to: (1) be center-based, (2) provide direct educational services to children, with a strong focus on cognitive and/or language development; (3) take place for at least $10 \mathrm{~h}$ per week for two months, and (4) serve the general population. Studies also had to have a comparison group. The authors identified 123 studies spanning five decades that met inclusion criteria; however, only 43 of these examined socio-emotional outcomes. The authors combined "social/emotional and anti-social outcome[s]" for analysis, including children's self-esteem, school adjustment, educational goals, aggression, and antisocial behaviors (p. 592). Results indicated that participation in ECE programs was associated with modest positive effects on children's social skills and school progress (unweighted mean $\mathrm{ES}=0.16$ for treatment versus control group analyses). 
These treatment effects were maintained over the course of longitudinal follow-up. Two instructional practices were positively correlated with socio-emotional gains among treatment group members: teacher-directed instruction and small-group learning.

D'Onise et. al. (2014) conducted a systematic literature review examining the effects of center-based preschool programs on health outcomes. They identified 13 studies that examined the effects of program participation on "social competence" between grades one and 11. Several of these studies utilized measures that assessed both positive social behaviors (e.g., cooperation, self-control) and problem behaviors (e.g., externalizing and internalizing problems, hyperactivity). D'Onise and colleagues reported that eight of the 13 studies identified beneficial effects of preschool participation on social competence, broadly construed, whereas six found no significant effects. Program duration and quality were not significantly associated with impacts on socio-emotional functioning.

\section{Studies not included in meta-analyses and reviews (Tables 2 and 4)}

Several additional studies have been published since the aforementioned meta-analyses and reviews on the relationship between early intervention and SEL. This notably includes the only peer-reviewed study on the relations between public prekindergarten participation and SEL that our review uncovered: Weiland and Yoshikawa's (2013) investigation of the Boston Public Schools prekindergarten program (Table 1). Boston Public School preschoolers were compared to control group members who had a variety of experiences during the prekindergarten year, ranging from familial care to center-based preschool programming. All participants completed performance-based and observational assessments of SEL across the preschool year. Results indicated that public prekindergarten participants exhibited significantly greater growth in emotion regulation and inhibitory control over time than the control group.

Several recent studies have also investigated the relations between multi-component ECE program participation and SEL. For example, Brown and Sax (2013) reported on the SEL of preschoolers attending an arts-integrated Head Start site, the Settlement Music School's Kaleidoscope Preschool Arts Enrichment program ("Kaleidoscope"). The Kaleidoscope site combined traditional early learning strategies with comprehensive arts programming (e.g., visual art classes, dance and creative movement, music). This programming drew from varied cultural traditions, and was designed to support children's creativity and emotional expression. Results indicated that Kaleidoscope participants exhibited significantly greater growth in both positive and negative emotion regulation over the course of the school year, compared to children attending a traditional Head Start site. These results underscore the potential value of multi-component programming (including arts enrichment) in promoting young children's SEL.

Several years later, Reynolds et. al. (2016) published an evaluation study examining the effects of the Child-Parent Center (CPC) program on SEL. The CPC program provides comprehensive, center-based educational and family support services to lowincome children between Preschool and third grade. Reynolds and colleagues' study utilized a quasi-experimental, matched-group cohort design which included 1724 children who attended CPC preschool, and a comparison group of 906 children who attended alternative prekindergarten programming. Teachers rated children's SEL across the preschool year using the observation-based Teaching Strategies Gold Assessment System 
(TSGOLD). Overall, teachers rated CPC participants as having significantly higher overall SEL (including self-regulation, sustaining positive relationships, and participating constructively in group situations) at the end of preschool than comparison group members (standardized mean difference $=0.44$ ). These results should be interpreted in the context of the non-randomized design and reliance on teacher ratings; however, they suggest that participation in public school-based ECE programs may enhance the SEL of low-income children.

Richardson et. al. (2017) also examined SEL in the context of the CPC program. Their study's intervention group included 1289 low-income children who attended CPC preschool in Chicago. The comparison group included 591 children who attended public preschool programs at matched school sites. Teachers rated children's SEL skills at three time-points throughout the preschool year using TSGOLD. Results indicated that teachers rated CPC participants as having significantly higher SEL school readiness than control group members. Positive impacts were detected for children who attended both the full- and half-day CPC programs, and for children from free-lunch eligible and Spanishspeaking families.

\section{Skills-based SEL interventions}

Discrete skills-based interventions to augment SEL are appealing in that they can be delivered by a teacher in the school setting, and generally require a finite investment of time, training, and resources. These types of interventions often target student competencies through a combination of indirect methods such as teacher skills augmented through professional development and strategies to alter classroom quality or parent training, and direct methods like didactic teaching and practice of socio-emotional and self-regulation skills.

It should be noted that many programs that may come to mind when discussing interventions focusing on social skills within preschool programs use measures of psychopathology outcomes (e.g., emotional or behavioral disorders; symptoms of AttentionDeficit/Hyperactivity Disorder or clinically significant conduct problems) as proxies for "socio-emotional competencies" and as such, were not included in this review for reasons of construct validity discussed above. Several studies were also excluded from the present review due to lacking a control group, having a cross-sectional design, or taking place before 1990. Few studies collected long-term follow-up data, and therefore in many cases sustained effects of skills-based interventions are unknown. Every effort was made to review a representative body of evidence for the programs described below; however, for several programs, we were only able to identify one empirical study that met our inclusion criteria.

\section{Reviews of skills-based SEL interventions (Table 5)}

While there have been several reviews of programs designed to reduce challenging and increasing prosocial behaviors, only two comprehensive, peer-reviewed systematic reviews focused on skills-based SEL programs for young children had been published by our cutoff (Barton et al., 2014; Joseph \& Strain, 2003). The scope of these reviews is somewhat broader than that of the present paper, as both included studies of interventions across the elementary years in addition to those targeting preschoolers, as well 
as interventions that focused primary on parents and parenting practices. However, both reviews did evaluate the evidence base for many programs relevant to the current review-namely, universal SEL skills-based programs designed to be implemented by teachers in preschool settings.

Both Joseph and Strain (2003; Fig. 1) and Barton et. al. (2014; Fig. 2) have published reviews of socio-emotional curricula. In both reviews, studies had to meet the following criteria: (a) intervention targeted socio-emotional and behavioral competencies, (b) intervention targeted children from birth to age five, (c) intervention had a published manual, and (d) findings were published in a peer-reviewed journal article. Figures 1 and 2 summarize the number of studies that the authors identified, and the criteria that they used to judge program efficacy and implementation success. In both studies, programs were rated as having high, medium, or low levels of empirical evidence.

The aforementioned reviews have served as a valuable and informative starting point for the present study's investigation of the current state of the research on skills-based SEL programs; however, the criteria for choosing studies in the present paper differed considerably. Specifically, several of the interventions evaluated in Joseph and Strain's (2003) and Barton et. al. (2014) reviews were last evaluated prior to 1990; were primarily focused on mental health, psychopathology, or antisocial behavior as opposed to socioemotional functioning; or were evaluated in kindergartners or older elementary school aged children. Overall, most studies did not evaluate SEL using independent observers or multiple raters, and long-term follow-up was rare.

Meanwhile, two recently published meta-analyses investigated the effects of skillsbased SEL interventions on young children's SEL (Luo et al., 2020; Murano et al., 2020). Luo et. al. (2020) meta-analysis investigated the impacts of classroom-wide social-emotional interventions (e.g., universal, skills-based interventions) on preschoolers' social, emotional, and behavioral functioning. They identified 30 studies which reported sufficient data to calculate effect sizes, and which examined intervention effects on social competence. Meta-analytic results indicated that classroom-wide interventions had moderate positive effects on social competence ( $g=0.42, z=5.77, p<0.001, k=34$ ), though there was significant heterogeneity in effect sizes across studies. Follow-up analyses indicated that interventions that incorporated family-focused programming had greater impacts on social competence than those that did not include family members. Meanwhile, in meta-analysis of 12 studies that examined emotional competence, Luo and colleagues reported that classroom-wide interventions had moderate positive effects on emotional competence ( $g=0.33, z=2.85, p=0.004, k=14)$. Interestingly, univariate moderator analyses did not identify linkages between most intervention variables (including dosage) and children's SEL outcomes.

In another recent meta-analysis, Murano et. al. (2020) examined the effects of both universal and targeted skills-based SEL interventions on preschoolers' SEL skills and problem behaviors. They identified 48 empirical studies which met their inclusion criteria, and reported that both universal and targeted interventions had moderate effects on children's social and emotional skills ( $g=0.34$ and $g=0.44$, respectively). Similar to the findings reported by Luo et. al. (2020), Murano and colleagues indicated that there was significant heterogeneity in effect sizes across studies, and that $83 \%$ of this heterogeneity was attributable to the specific intervention implemented. They also reported that 


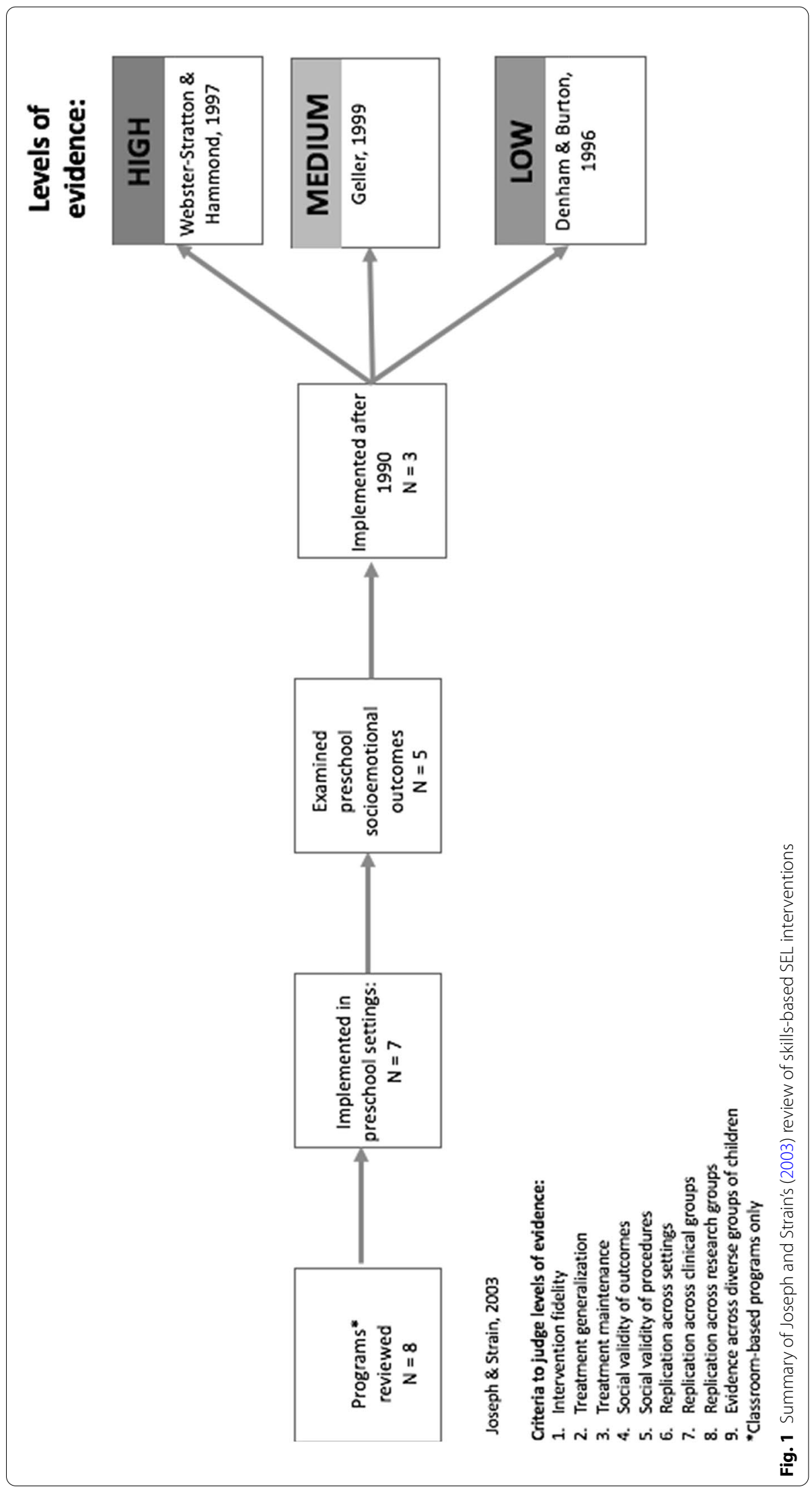




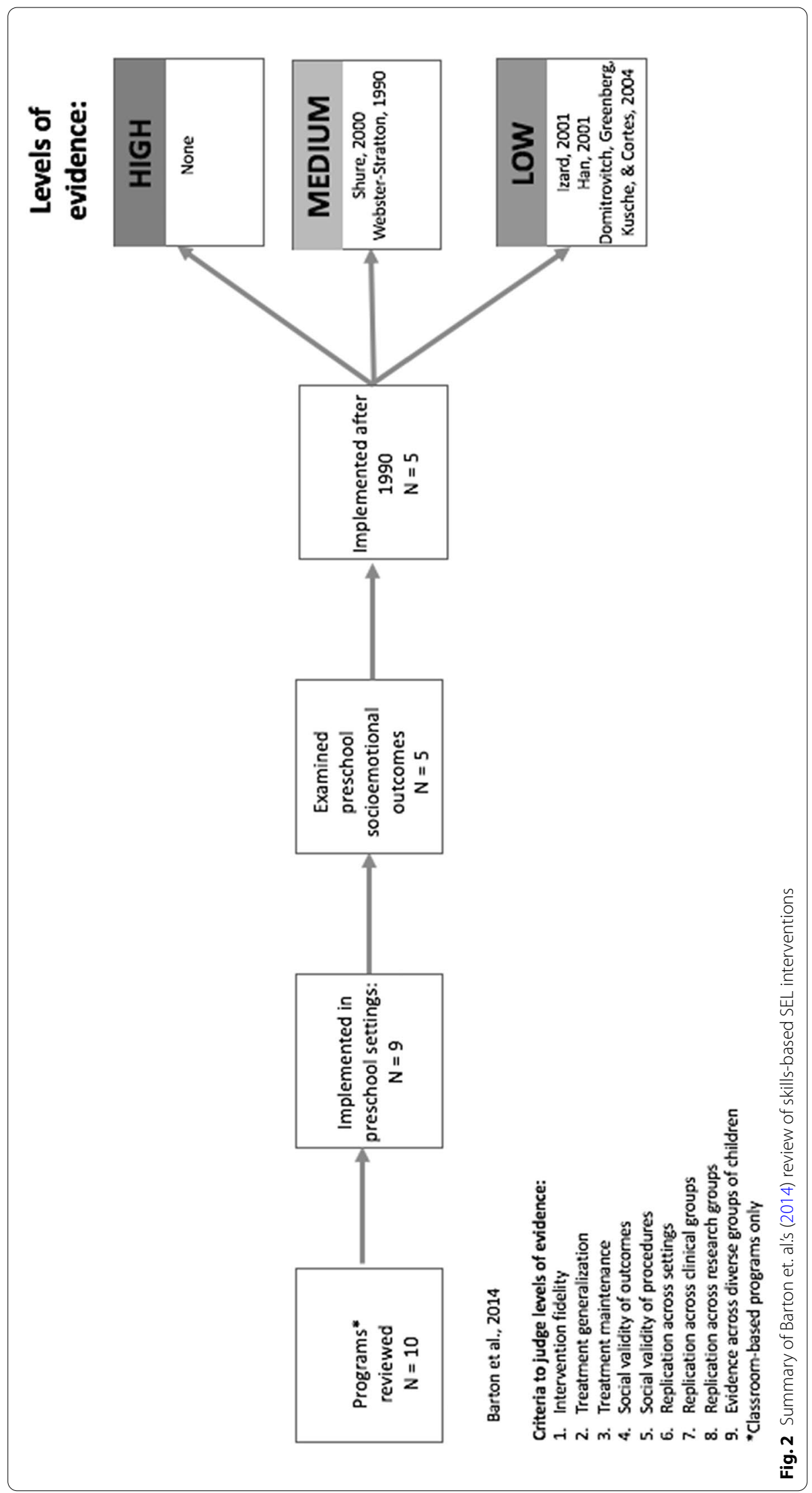


interventions that included family members had stronger impacts on children's SEL than those that did not include family members-also in keeping with Luo and colleagues' results.

Taken together, Luo et. al. (2020) and Murano et. al. (2020) meta-analyses support the effectiveness of skills-based SEL interventions in promoting young children's SEL. Their results indicate that both universal and targeted interventions can be beneficial, and that interventions that operate at multiple social-ecological levels tend to be most effective. Building on these findings, we will now summarize the empirical evidence bases for several SEL skills-based interventions in depth. Our intention is to describe several highquality interventions, as a way of highlighting exemplary research as well as conceptual and methodological issues for future researchers to address.

\section{Child and teacher-focused skills-based interventions}

This group of interventions provides a teacher-training component combined with a child curriculum consisting of discrete, manualized lessons on SEL topics. These curricula often take the form of teaching didactic SEL skills instruction to children, typically in group-based settings in the context of Head Start and other publicly funded preschool programs. Due to space constraints, three exemplar programs will be discussed in detail below. Table 6 describes additional skills-based interventions meeting our criteria.

\section{PATHS}

One child and teacher-focused skills-based intervention is the Promoting Alternative Thinking Strategies (PATHS) curriculum (Domitrovich et al., 2004). PATHS is one of the most extensively evaluated SEL programs for young children (Arda \& Ocak, 2012; Domitrovich et al., 2007; Hamre et al., 2012; Hughes \& Cline, 2015; Stefan \& Miclea, 2012). The developers describe PATHS as based on the Affective-Behavioral-Cognitive-Dynamic (ABCD) model of development (Greenberg \& Kusche, 1993), which "suggests that emotional development is an important precursor to other cognitive and language skills and that the successful development of emotion knowledge and regulation is foundational to the development of the broad spectrum of social competencies described previously as central to school success (Hamre et al., 2012, p. 811)." Although implementation appears to vary slightly by site and evaluation team, the curriculum generally consists of several dozen lessons, delivered once per week by Head Start preschool teachers during "circle time," and focuses on emotion knowledge, regulation, prosocial skills, and problem-solving. The intervention also often includes extension activities that were intended to generalize the weekly lessons and to foster an environment that would encourage children's use of socio-emotional skills. Teachers are generally provided with support, ranging from access to a website with examples of teachers implementing PATHS to ongoing site visits and consultation from designated PATHS coordinators. Implementation fidelity has also been monitored in a variety of ways, including via the site visits or through submission and coding of videos. Studies utilized a range of tools for assessing outcomes, including direct assessment, observation, and parent and teacher reports of emotion knowledge, prosocial and problem behavior, and attentional skills. Evaluators generally reported medium effect sizes, ranging from 0.20 to 0.50 , across socio-emotional and behavioral domains. However, as in many of the studies 
included here, parents and teachers in all of the evaluations were not blind to the intervention condition, which could have biased ratings.

Stefan and Miclea (2012) evaluated the implementation of a program that they called "Fast Track," in which PATHS was the SEL intervention, in a preschool population in Romania. In addition to teacher training, they utilized a parent component, which was delivered via group and individual training sessions, and focused on positive discipline strategies and increasing parents' knowledge of how to support their child's social and emotional development. The authors found medium to large intervention effects for all outcomes. Children in medium and high-risk subgroups appeared to benefit more from the intervention even when controlling for baseline differences on tasks. This study is distinguished by the fact that the authors conducted follow-up assessments three months after the post-intervention data was collected, and found that intervention effects were maintained for both social and emotional competencies. However, there was no direct observation of child classroom behaviors, and as teacher, parent, and child components were delivered concurrently, mechanisms of effects are unknown.

\section{Head Start REDI}

Another Head Start-based program, Head Start Research-based, Developmentally Informed (REDI), which uses PATHs to target socio-emotional skills in the context of a broader program intended to enhance both social competence and literacy in preschoolers, has been the subject of several studies with long-term follow-up (Bierman et al., 2014; Bierman, Domitrovich, et al., 2008; Bierman, Nix, et al., 2008; Nix et al., 2016). Initial investigations of the REDI program on preschoolers found small to medium effect sizes for social competence and executive functioning outcomes (Bierman, Domitrovich, et al., 2008; Bierman, Nix, et al., 2008). Results of a 1-year follow-up on children in Head Start REDI as preschoolers compared to those who had attended "usual practice" Head Start indicated sustained direct effects for social problem-solving $(d=0.40)$ and parent and teacher-rated aggressive behaviors $(d=-0.20$ and $d=-0.25$, respectively) for kindergarten children who were in REDI as preschoolers, with effect sizes at the 1-year follow-up equal to or larger than those at the end of the intervention year. Teacher-rated social competence was also significantly higher for intervention students $(d=0.26)$, but only for those in kindergarten at schools where overall student achievement was low (Bierman et al., 2014). Several years later, Bierman et. al. (2017) combined the samples of the two previous randomized trials to investigate the sustained effects of REDI, three years post-intervention. Intervention group participants received either the REDI Classroom Program (REDI-C), or both REDI-C and the REDI-Parent home visiting program (REDI-P). Comparison group members attended standard Head Start programming. Results indicated that REDI-C graduates exhibited significantly higher classroom participation $(d=\sim 0.25)$, social competence $(d=\sim 0.25)$, and student-teacher relationships $(d=\sim 0.4)$ in second grade relative to the comparison group. Children who had received both REDI-C and REDI-P exhibited higher perceived social competence $(d=\sim 0.75)$ compared to children who had only received REDI-C.

Taken together, these studies provide strong evidence that the PATHS curriculum, when implemented with fidelity, can effectively increase the SEL competencies of 
preschoolers both within the context of the literacy-promoting REDI intervention and when used independently.

\section{Kindness Curriculum}

The Kindness Curriculum, a mindfulness-based intervention designed to increase empathy, prosocial behavior, and self-regulation in preschoolers, has been evaluated in two empirical studies meeting our criteria (Flook et al., 2015; Poehlmann-Tynan et al., 2016). In both evaluations, the 10-h training was delivered to preschoolers over the course of 12 weeks via stories, music, and movement. The program emphasizes kindness, emotion regulation, and attentional capacity. Flook et. al. (2015), in the initial study, found via teacher ratings and direct assessment that the intervention group showed greater improvements across domains of social competence $(d=0.26-0.29)$ compared to the control group, in addition to significant effects for cognitive flexibility $(d=0.43)$ and delay of gratification $(d=0.23-0.37)$. Children who were initially lower in social competence and executive functioning skills evinced larger gains in social competence.

Poehlmann-Tynan et. al. (2016) also found promising effects when investigating this curriculum in economically disadvantaged preschoolers and assessed prosocial behavior (e.g., empathy and compassion), self-regulation, and executive functioning via direct assessment and observation. They found that the children in the $\mathrm{KC}$ intervention group increased their capacity for self-regulation and attention (partial $\eta^{2}=0.26-0.33$ ) relative to the control group; however, unlike Flook, they found no changes in observer-rated or directly assessed prosocial behaviors. The positive effects, however, persisted at a 3-month follow-up assessment. It is important to note that the curricula were delivered by "experienced mindfulness instructors." The evidence base for effectiveness and scalability of the $\mathrm{KC}$ intervention in a preschool setting would be strengthened by an investigation of the program delivered by classroom teachers.

\section{Skills-based interventions incorporating parent involvement}

This group of interventions supplements skills-based interventions for children and/ or teachers with parent involvement initiatives. Due to space constraints, three exemplar programs will be discussed in detail below. Table 6 describes additional skills-based interventions.

\section{The RECAP intervention}

Han et. al. (2005) have published the only study to date evaluating the Reaching Educators, Children, and Parents (RECAP) program in preschoolers. The study aimed to enhance preschoolers' problem solving and social skills and also attempted to incorporate a parenting component. The program included curricular and behavior management components, provided teachers with weekly trainings and ongoing consultations, and offered a bi-weekly parent group. Study participants were primarily low-income 4 to 5-year-old children in public prekindergarten classrooms. The evaluators collected parent- and teacher-report of internalizing and externalizing behaviors and social skills. They found no changes in children's parent-rated functioning over time. However, they did find significant improvements for intervention group participants on teacher-rated total social skills $(F[1,144]=5.73, p<0.05)$, cooperation $(F[1,144]=3.99, p<0.05)$ and 
assertion $(F[1,144]=7.12, p<0.01)$. Notably, parent group attendance was extremely low, and as such, the effectiveness of the parent component of this intervention was not able to be evaluated. Other limitations included the self-report nature of the outcome measures, and baseline group differences in teacher-reported levels of problem behaviors and skills, and on family income.

\section{Making choices and strong families (Conner \& Fraser, 2011)}

The Making Choices program is one of the few studies of an SEL skills-based curriculum for preschoolers that included a successfully implemented parenting component. The SEL-focused component, Making Choices, is a manualized program with theoretical bases in social information processing, designed for preschool-aged children. The program was delivered twice weekly for 14 weeks with the aim of strengthening social information processing, emotion regulation skills, and prosocial interactions with peers. The parent training component, Strong Families, had distinct but complementary goals, including increasing positive parent-child interaction patterns and decreasing coercion. The study sample was drawn from a group of part-day preschool centers, and the comparison group received typical Head Start services. Investigators directly assessed child-level competencies (e.g., academic competence, achievement motivation, social competence, peer acceptance, depression/anxiety and aggression/hostility) and family and child functioning. Results revealed significant effects for all child competencies $\left(F[20,46]=3.05, p<0.001 ; r^{2}=0.35\right)$, with higher gain scores among intervention group participants, as well as positive changes in caregiver behaviors $(F[10,56]=6.88$, $p<0.001 ; r^{2}=0.36$ ). While these outcomes are promising, the sample sizes are quite small, and about $35 \%$ of eligible families moved or otherwise became ineligible before assignment to a treatment group. No follow-up or replication studies in preschoolers have been conducted to date, and as the interventions were delivered together, it is unclear if one or both was driving effects.

\section{Incredible Years (IY)}

The Incredible Years program was originally designed as a treatment for children with Oppositional Defiant Disorder or Conduct Disorder diagnoses (Webster-Stratton, 1990), with theoretical bases in social learning theory and the effects of adult-child interaction processes in child behavior. The program has been adapted for use with several age groups and populations, including as a classroom-based prevention program aiming to augment socio-emotional skills and decrease problem behaviors in preschoolers. Of particular interest to the present review are the IY Teacher Classroom Management Training (TCM), the IY Child Program: Dinosaur Classroom Curriculum, and the IY Parent Program used in conjunction with the teacher or child programs. The IY programs, implemented as universal prevention programs in preschool-aged children, have been evaluated by Webster-Stratton and colleagues (Webster-Stratton, 1998; Webster-Stratton \& Reid, 2004; Webster-Stratton et al., 2001, 2008). Several independent evaluations of IY programs have also been conducted; however, many specifically evaluated children with high levels of disruptive or oppositional behaviors in the preschool setting (e.g., BakerHenningham et al., 2012), did not utilize a control group (e.g., Shernoff \& Kratochwill, 
2007), or did not measure SEL outcomes (e.g., Williford \& Shelton, 2008). As such, they did not meet our inclusion criteria.

Webster-Stratton et. al. (2001) evaluated IY TCM program in combination with the Parent Training as a universal prevention program in Head Start. The intervention group parents and teachers participated in the IY programs, and the control group received usual practice Head Start services. The teacher training consisted of $36 \mathrm{~h}$ of training on classroom management, child development, and promotion of prosocial and reduction of antisocial behaviors. The parent training had similar content, adapted for the home context and focused on reducing coercive discipline and increasing positive parenting practices. Teachers and parents in the intervention group evinced more positive practices, while children engaged in more prosocial behavior and were rated as more socially competent. Specifically, $71 \%$ of intervention group children rated as having problems with social competence at baseline fell in the normative range at the end of school, compared to $36.6 \%$ of the control group children initially rated as lower in social competence $\left(\chi^{2}[1,26]=4.12, p<0.04\right)$.

Webster-Stratton and colleagues have also evaluated the IY TCM program in combination with the Dinosaur School curriculum in Head Start settings (Webster-Stratton et al., 2008). Children received 30 bi-weekly lessons promoting socio-emotional skills, problem-solving, self-regulation, and school behavior over the course of a year, communicated via vignettes, small-group activities, puppets, and games. Teachers participated in $28 \mathrm{~h}$ of workshops focused on classroom management and promotion of socio-emotional competence, spread out over four months. A research staff-member led lessons alongside the classroom teacher to ensure implementation fidelity. Outcomes were measured via classroom observations, as well as direct assessment of competencies such as problem-solving skills and emotion knowledge. The authors reported that teachers in the intervention were more likely to use teaching strategies to promote SEL (e.g., teaching prosocial behavior, problem-solving, shaping peer play, encouraging feelings language, and promoting social competence) in intervention group teachers $(d=0.96)$. The intervention was also associated with higher levels of teacher-reported child social competence and self-regulation (effect sizes not reported), particularly for students with low levels at baseline, as well as improvements in problem-solving $\left(\eta^{2}=0.41\right)$ and feelings knowledge $\left(\eta^{2}=0.14\right)$.

Overall, the evidence base for the Incredible Years intervention is encouraging; however, evaluation methodology has been inconsistent and further investigation is needed to determine efficacy of the program as a universal prevention strategy for preschoolaged children.

\section{Teacher-focused skills-based interventions}

This group of interventions provides training and other forms of professional development to teachers, with the aim of improving teacher-child interactions and children's socio-emotional functioning.

\section{Pyramid Model}

The Pyramid Model for Promoting Young Children's Socio-Emotional Competence (the "Pyramid Model"; PM) is a professional development intervention that includes 
research-informed practices for promoting healthy socio-emotional development and high-quality relationships between caregivers and children. PM practices include universal strategies for teachers to promote family engagement and children's peer social skills, as well as individualized interventions strategies for children exhibiting challenging behaviors. These practices are taught through multi-day workshops and implementation guides. Teachers also receive classroom materials (e.g., puppets, books) for implementing the practices.

Hemmeter et. al. (2016) conducted a cluster-randomized controlled potential efficacy trial to evaluate the effects of classroom-wide implementation of PM on teacher practices and child outcomes. Teachers in the intervention group participated in the initial PM workshops, and subsequently received weekly individualized coaching, which included in-classroom observation. Results indicated that children whose teachers participated in PM had higher teacher-rated social skills at post-test than children whose teachers did not participate in PM $(d=0.43)$. These results are promising, but should be interpreted with caution given the study's reliance on teacher ratings of children's social skills.

\section{Foundations of Learning (FOL)}

Foundations of Learning is a professional development intervention that combines teacher training and mental health consultation. FOL teachers participate in workshops on proactively supporting positive behavior and managing challenging behaviors in the classroom, and on personal stress management. They also receive weekly classroom-level mental health consultation, and individualized mental health consultation as needed for children exhibiting persistent challenging behaviors. FOL's teacher training component is adapted from the Incredible Years curriculum; the intervention is also based on the previously discussed, smaller-scale Chicago School Readiness Project (CSPR) intervention.

Morris et. al. (2013) conducted a cluster-randomized controlled study to evaluate the effects of FOL on preschool teacher practices and child outcomes. Results revealed positive impacts on teachers' ability to manage challenging behaviors and promote a positive emotional climate in the classroom. At the child outcome level, results indicated that children whose teachers participated in FOL exhibited less problem behaviors (e.g., peer and teacher conflict, as rated by trained observer) than children whose teachers did not participate in FOL. However, no significant effects on either observer- or teacher-rated positive social behavior (e.g., communication, sociability, compliance) were detected between groups. Positive intervention effects on children's approaches to learning (e.g., self-control, focus, and participation in classroom activities) were detected at the trending level $(p<0.10)$.

\section{VIDA}

Jensen et. al. (2017) evaluated the VIDA (a Danish acronym for Knowledge-based efforts for socially disadvantaged children in daycare) intervention, which aims to augment socio-emotional functioning in preschool children by altering their social context. The primary mode of change is via teacher training to improve the preschool environment. Intervention teachers attended 17 full days of training over the course of 2 years, 
gaining theoretical knowledge about child development and the bioecological system, encouraging reflection, enhancing communication with students, and requiring teachers to use the training to design their own activities focused on socio-emotional skills (e.g., improving friendships, managing conflict). Jensen et. al. (2017) explain:

"The initial step of the teachers' learning process takes place as a top-down process that presents participants with predefined topics [...] Through reflection, everyday experiences are related to the research-based knowledge and the teaches are using this to change their practice. The process transforms what was initially top-down, course-based theoretical knowledge into bottom-up, practice-oriented teacher learning and innovation" (p. 28).

Teacher ratings revealed a trending effect of the intervention on prosocial behavior. Further evaluation of the program is warranted, and results may not be generalizable due to the high dosage of the program. It is also unclear whether students retained the same teacher over the course of the intervention, which could be an important confounding factor. Finally, conclusions would be strengthened by measurement of SEL outcomes via more diverse tools, as the only outcome measure in the present study was teacher-reported.

\section{Discussion}

\section{Comparing program types}

\section{General public prekindergarten programs}

Access to public prekindergarten programs has expanded dramatically in the United States over the last several decades, with approximately one-third of 4-year-old children enrolled in state-funded programs in 2017 (National Institute for Early Education Research [NIEER], 2018). These programs have historically focused on enhancing children's pre-academic skills (e.g., language, numeracy), but have also increasingly targeted SEL.

Participation in public prekindergarten may enhance SEL by several mechanisms. For example, high-quality teacher-child relationships have been linked to improvements in children's SEL (Merritt et al., 2012). Prekindergarten participation also provides children with consistent opportunities for socialization with peers and social skill practice. Finally, improvements in children's academic and cognitive skills at the individual and classroom levels may also contribute to improvements in SEL over time through spillover effects. For most children, this may be sufficient, but it is important to evaluate whether public prekindergarten programs can exert significant and sufficient benefits on children's SEL, or whether more targeted SEL services are needed.

Our review identified only one peer-reviewed study examining the effects of a general public prekindergarten program (Boston Public Schools) on SEL (Weiland \& Yoshikawa, 2013). Major strengths of this study include the socioeconomically diverse sample, utilization of performance-based measures of SEL skills, and examination of subgroup effects by race/ethnicity and free/reduced lunch status. Effect sizes on cognitive inhibitory control and emotion recognition were small, but statistically significant. The authors posited a "spill-over" hypothesis to explain program impacts on inhibitory control. The results of this study suggest that high-quality general public prekindergarten 
programming may have a positive impact on children's SEL, but that children who exhibit delays in developmentally appropriate SEL skills may benefit from more targeted intervention. This finding is consistent with a significant body of research suggesting that children with higher needs tend to benefit more from early childhood intervention (Reynolds et al., 2011; Washington State Institute for Public Policy, 2014). Nonetheless, given that prekindergarten programs vary widely by school district, there is a need for additional studies in this domain. There is a particular need for studies examining the differential contributions of various program components. This is an important consideration in Weiland and Yoshikawa's (2013) study given the unusually high quality of the Boston Public Schools prekindergarten program, which includes equal educational requirements and pay scale for teachers from prekindergarten through high school, a research-based academic curriculum and a district-designed teacher coaching system.

\section{Multi-component ECE programs}

Multi-component ECE programs (e.g., Head Start, the Child-Parent Center (CPC) Program) typically provide a more comprehensive array of academic and family support services than general public prekindergarten programs. These programs also commonly prioritize enrollment of low-income children, who often lag behind in acquisition of pre-academic and SEL skills. Previous research has demonstrated that participation in these programs is most beneficial for children with the lowest levels of skills and the highest levels of psychosocial risk at program entry (Karoly \& Bigelow, 2005; Reynolds et al., 2007). Hypothetically, multi-component programs may enhance children's SEL by addressing risk and protective factors at multiple social-ecological levels. This is accomplished through a variety of means, from comprehensive academic curricula to wraparound family and social services.

The present review identified several literature reviews and meta-analyses examining the effects of multi-component prekindergarten programs on SEL. Many of these studies constructed outcome variables that combined SEL and mental health outcomes; thus, their results should be interpreted with significant caution. Nonetheless, aggregate results indicated that program participation was associated with small to moderate gains in SEL compared to comparison group members, with multiple studies reporting that children affected by the highest psychosocial risk exhibited the greatest gains. Findings on the impact of program duration were mixed. One meta-analysis reported that didactic instruction and small-group learning were positively associated with participants' SEL gains (Camilli et al., 2010), suggesting that a balance of teacher-directed instruction, and child-initiated and small group activities may be beneficial for SEL. Meanwhile, two additional studies indicated that participation in the CPC prekindergarten program was associated with moderate enhancements in SEL for low-income children from diverse backgrounds (Reynolds et al., 2016; Richardson et al., 2017).

This body of research is small, but suggests that multi-component programs hold promise for promoting SEL. Notably, most studies did not examine the differential impacts of various program components on SEL (e.g., professional development, curricula, classroom structure), making it difficult to determine whether SEL benefits were driven by the overall combination of program components or a small number of "active ingredients." 


\section{Skills-based SEL interventions}

There is no shortage of skills-based SEL interventions for young children; however, they vary widely in scope, focus, size, theoretical foundations, and quality of the evidence base. Generally, such programs are based on manualized curricula, and are designed to supplement or enrich typical preschool programs. This type of intervention can be efficient, cost-effective, and scalable. For example, schools are not required to adopt an entirely new multi-component educational program, and the explicit targeting of specific SEL skills through games, songs, vignettes, role playing and modeling, and didactic teaching, and/or through teacher professional development and parent coaching can be a developmentally appropriate, engaging, and effective way to reach preschool-aged children. All of these factors likely contribute to the abundance of skills-based programs meeting our criteria for review in the present article.

We first identified two previously published reviews of skills-based SEL interventions (Barton et al., 2014; Joseph \& Strain, 2003). Importantly, the authors state that they only reviewed studies of programs used in "at-risk" populations or with children demonstrating behavioral challenges. This review, in contrast, intentionally focused on empirical studies of the effectiveness of programs (some of which also appeared in the aforementioned reviews) in a general classroom setting. We felt that this was crucial, as all children can benefit from SEL skills training, and expanding the use of high-quality universal programs can help to shift the paradigm from pathologizing children with SEL "deficits" to normalizing and encouraging SEL growth for all children. Strain of the 2003 review was also an author on the 2014 paper, and as such, both papers used the same criteria for inclusion and assessment of quality. Ultimately, their conclusions were quite mixed. Of the SEL-focused programs evaluated in preschool settings after 1990s, the authors of both reviews identified one program with "high" levels of evidence, three with "medium" levels of evidence, and four with "low" levels of evidence.

We also identified two recently published meta-analyses, which examined the evidence base on skills-based SEL interventions for preschoolers (Luo et al., 2020; Murano et al., 2020). These meta-analyses indicated that both universal and targeted skills-based interventions had significant, moderate effects on preschoolers' SEL skills. Both studies also reported limited evidence for moderating effects, and noted that interventions with family components were more effective than those that did not include family members. These results provide the strongest evidence to date that skills-based interventions can support the SEL of young children, including those affected by sociodemographic risk factors.

Overall, the results of the aforementioned systematic reviews and meta-analyses are consistent with the present review. We found considerable variation in delivery methods, assessment methods, and outcomes across interventions, in addition to variation in the use of control groups, random assignment, outcome measurement, follow-up, and other crucial elements of empirical research. While some programs were explicitly grounded in theory (some in social learning theory and principles of social information processing; others in more broadly defined developmental, self-regulation, and systems theories), effect sizes varied considerably within and across programs.

Despite these challenges, several programs with strong theoretical bases have been evaluated with large sample sizes, random assignment, multiple sources of outcome 
assessment, and short-term follow-up, and as such, it is our cautious conclusion that skills-based programs can be an effective way to augment SEL skills in young children. Whether such programs are the optimal way to augment these skills (as opposed to the other approaches examined in the present paper) remains to be seen. The next step is to investigate impacts in well-designed quasi-experimental or randomized designs, while establishing and maintaining construct validity around SEL and ensuring that programs can be effectively delivered in real-world settings.

\section{Synthesis: three intervention approaches}

There are clear benefits and drawbacks to the three intervention approaches that were reviewed in the present paper. From a developmental perspective, there is considerable evidence for the use of multi-component ECE programs, which aim to promote holistic development by enhancing protective factors and reducing risk factors at multiple social-ecological levels. However, there is also promising evidence for several skillsbased SEL programs, which have the benefit of facilitating adoption and implementation within existing frameworks.

Decades of developmental research have indicated that sensitive, responsive caregiving is an essential catalyst for healthy development in infancy and early childhood (Ainsworth et al., 1978; Landry et al., 2000). Given this evidence, prekindergarten programs that facilitate high-quality teacher-child relationships (e.g., via professional development, small class sizes) and safe, stable learning environments are likely to exert positive impacts on children's SEL. Multi-component ECE programs, which typically include interventions at multiple levels of children's social ecologies, may have an advantage in this domain over skills-based SEL programs. To this end, multi-component ECE programs fall under the category of promotion programming, as identified by the Center on the Social and Emotional Foundations for Learning (CESFEL; Duran et al., n.d.). Promotion programming includes interventions, practices, and policies that ensure that all children are receiving high-quality caregiving and education, which will facilitate developmentally appropriate SEL. Universal skills-based interventions that are offered to all children in a classroom may also fall under the promotion realm. Skills-based interventions may also be offered at the prevention level (Duran et al., n.d.). Programming at the prevention level provides targeted SEL support services for children with emergent SEL challenges, with the goal of addressing these challenges before they develop into more serious psychopathology. Offering skills-based SEL interventions at the prevention level for indicated populations may be more cost-effective than universal implementation; however, this strategy requires a screening process for identifying children at risk. School-based, skills-based SEL interventions may not be sufficient for children with more serious SEL deficits and/or clinical psychopathology; this population may benefit from more intensive intervention services in a mental health setting (Duran et al., n. d.).

Beyond program efficacy, several other factors must be considered when selecting an intervention, including cost, ease of implementation, and scale-up potential. Substantial financial resources and infrastructure are required to implement multi-component ECE programs and public prekindergarten programs; however, cost-benefit analyses have indicated that these initial investments may yield significant returns over time. For example, Heckman and colleagues have estimated that high-quality ECE programs can 
produce financial returns of as much as $13 \%$ per annum (Garcia et al., 2016). Longitudinal research has also demonstrated that ECE programs can exert enduring benefits on many aspects of wellbeing (e.g., Consortium for Longitudinal Studies, 1983; Reynolds \& $\mathrm{Ou}, 2011)$.

Researchers have also investigated the monetary value of interventions that specifically target SEL, and have found that such programs can yield substantial economic returns (e.g., Belfield et al., 2015). Studies indicate that these savings stem from improved functioning among program graduates, including reductions in substance abuse and increases in earnings, often mediated through variables such as educational attainment and self-esteem (Araujo \& Lagos, 2013; Klapp et al., 2017). Aspects of SEL often characterized as "self-control" variables (e.g., executive functioning, self-regulatory skills) may also help to explain returns on investment in SEL programs. Childhood self-control has been found to predict costly outcomes, including physical health, substance use, income, and crime in adulthood (Moffitt et al., 2011). Finally, several studies have investigated a subset of SEL-informed intervention programs which narrowly focus on reducing delinquency and substance use. They note that these programs tend to target a small subset of SEL-related skills (e.g., impulse control) and can yield cost savings by reducing involvement in the criminal justice system (Miller \& Hendrie, 2008).

\section{Future research directions}

The present paper aimed to review the highest quality literature available on the relationship between prekindergarten programs and SEL. Our review indicated a number of common methodological issues which should be addressed in future work.

\section{Definitions and measurement of SEL}

Evaluation studies of prekindergarten programs have typically examined cognitive and academic outcomes, with few studies investigating impacts on children's SEL. Meanwhile, studies that have examined socio-emotional outcomes have typically focused on maladaptive behaviors and psychopathology (e.g., internalizing and externalizing symptoms). This is problematic, given that prekindergarten programming is not primarily intended to prevent or treat psychological symptoms. Rather, prekindergarten programs are designed to promote acquisition of developmentally appropriate skills. As such, researchers should carefully attend to construct validity by: (a) clarifying whether they are measuring psychopathology outcomes, SEL, or both; and (b) specify the SEL domains they are investigating, how they are operationalizing them, and how they are tracking growth in SEL competencies over time. At the broader field level, efforts must also be made to develop consensus on critical issues related to SEL measurement, concepts, and dimensions of relevance. While the work of CASEL (2012) and others has provided some clarity on these issues, researchers continue to use a wide array of labels for SEL phenomena (e.g., SEL, social competence, wellbeing, self-regulation) without clear definitions or parameters. Many studies have also stated that they are investigating "SEL" or similar phenomena, while solely utilizing outcome measures that assess psychopathology. Developing consensus on these issues will help to ensure construct validity, and also enable more rigorous comparative evaluations of different interventions. 
When examining all three types of interventions addressed in this review, it is also important for researchers to consider other potentially salient program components. For example, curriculum type, parent involvement, timing and duration of SEL components, and teacher and student supports that are not necessarily explicit components of the SEL training may all affect children's outcomes.

\section{Cultural considerations}

The present review indicates that relatively few studies have carefully attended to potential differential impacts of prekindergarten interventions on SEL for children from diverse cultural backgrounds. Research examining whether and how interventions impact the SEL of different subgroups of children could inform efforts to tailor interventions to the needs of specific populations.

Researchers should also carefully select assessment measures that are appropriate for use with multicultural populations. The present review indicates that most previous studies have utilized deficits-focused outcome measures (e.g., assessing the effects of intervention on psychopathology symptoms). Moving towards strengths/skills-focused outcome measures (e.g., assessing the effects of intervention on developmentally and culturally appropriate SEL skills) will likely increase the cultural sensitivity of research in this domain, and help to ensure that children from non-dominant cultures are not being improperly identified as having SEL deficits.

\section{Informants}

Our review revealed that numerous studies relied on non-blinded, single-informant reports of SEL outcomes-typically, reports from classroom teachers who were delivering interventions. Previous research has demonstrated the importance of utilizing multiple informants to minimize reporting bias (Totura et al., 2009). For example, surveying both teachers and parents can provide a more nuanced perspective on children's SEL skills in multiple environments (school and home). The use of trained observers or performance-based measures may also yield unique information about children's functioning.

\section{Control groups}

Our review identified and excluded a number of program evaluation studies that lacked control/comparison groups. The absence of control groups makes it impossible to determine whether changes in children's SEL are due to program participation as opposed to other factors like developmental maturation. It is essential that future studies include well-defined control/comparison groups so that program impacts can be adequately estimated.

\section{Measurement of multiple intervention components}

Several of the skills-based programs had professional development, didactic child skills, and parent-focused aspects, with little investigation of differential impacts of each program element or mechanism. The issue of mechanisms is not confined to multi-pronged interventions; rather, none of the programs reviewed analyzed how child-focused programs transmit positive impacts to the outcomes of interest. Future research should 
determine which and how specific aspects of programs (e.g., didactic instruction in problem-solving and conflict resolution; teacher-facilitated emotion recognition and expression) impact knowledge and behavior.

\section{Implementation fidelity}

We identified relatively few studies that provided information about implementation fidelity. Fidelity measurement is essential to accurately estimate program impacts. Fidelity measurement can also provide important information about whether program scaleup is feasible, or whether adaptations are needed to increase the program's practicality or cultural relevance (e.g., replacing doctoral-level clinicians with trained laypeople).

\section{Longitudinal follow-up}

Our review revealed a paucity of studies examining the longitudinal impacts of prekindergarten programs on SEL. Studies that did include multiple time-points rarely continued past early elementary school. This is an important limitation that raises questions about the stability of program impacts on SEL over time. Moving forward, there is a need for longitudinal studies that include pre-program assessments of baseline SEL, and that investigate participant outcomes through the school years and beyond.

\section{Conclusions and implications}

Interest in scalable strategies for enhancing children's SEL has grown steadily since the 1970s, when Edward Zigler argued that promoting 'social competence' should be the primary aim of early childhood interventions (Zigler \& Trickett, 1978). During the 1990s and 2000s, the development of the interdisciplinary SEL framework spurred additional research and policy initiatives in this domain. Numerous skills-based interventions have been developed for use in early care and education settings (Tables 5, 6), and general public prekindergarten programs and multi-component interventions have also demonstrated impacts on SEL (Tables 2, 3, 4). These developments are promising; however, moving forward it is essential that stakeholders define and measure SEL in ways that are consistent, developmentally appropriate, and culturally sensitive. Collaboration among diverse groups of stakeholders (e.g., community-based researchers, policymakers, parents, and early childhood leaders) will be essential to accomplishing these aims.

Finally, investments should be made into efforts to support children's SEL at multiple ecological levels, from home- and school-based interventions to public policies that support healthy development. Specifically, early childhood educators should place SEL skills alongside literacy and numeracy skills as an important part of a balanced early childhood curriculum. Policymakers, parents, and early childhood leaders can assist teachers in implementing SEL interventions or infusing SEL into existing programming by advocating for increased funding and materials for these efforts.

Acknowledgements

Not applicable.

Authors' contributions

The manuscript was co-conceptualized by $\mathrm{CM}, \mathrm{AG}$, and AJR. CM and AG conducted the literature review and were the major contributors in writing the manuscript. AJR edited the manuscript. All authors read and approved the final manuscript. 


\section{Funding}

CMR's work on this manuscript was funded by a Doris Duke Fellowship for the Promotion of Child Well-Being. The views and findings presented herein do not necessarily reflect the views of the Doris Duke Fellowship.

\section{Availability of data and materials}

Data sharing is not applicable to this article as no datasets were generated or analyzed during the current study. Please contact the authors with any questions.

\section{Declarations}

\section{Competing interests}

The authors declare that they have no competing interests.

\section{Author details}

${ }^{1}$ Division of Developmental Medicine, Brazelton Touchpoints Center, Boston Children's Hospital, Harvard Medical School, 1295 Boylston St., Suite 320, Boston, MA 02115, USA. ${ }^{2}$ Department of Pediatrics, University of California San Francisco, San Francisco, USA. ${ }^{3}$ Institute of Child Development, University of Minnesota, Minneapolis, USA.

Received: 2 April 2020 Accepted: 10 May 2021

Published online: 22 May 2021

\section{References}

Ainsworth, M. D., Blehar, M., Waters, E., \& Wall, S. (1978). Patterns of attachment: A psychological study of the strange situation. Lawrence Erlbaum.

Araujo, P., \& Lagos, S. (2013). Self-esteem, education, and wages revisited. Journal of Economic Psychology, 34(C), 120-132. https://doi.org/10.1016/j.joep.2012.12.001

Arda, T. B., \& Ocak, S. (2012). Social competence and promoting alternative thinking strategies_PATHS preschool curriculum. Educational Sciences: Theory \& Practice, 12(4), 2691-2698.

Baker-Henningham, H., Scott, S., Jones, K., \&Walker, S. (2012). Reducing child conduct problems and promoting social skills in a middle-income country: Cluster randomised controlled trial. British Journal of Psychiatry, 201, 101-108. https://doi.org/10.1016/j.joep.2012.12.00110.1192/bjp.bp.111.096834

Barton, E. E., Steed, E. A., Strain, P., Dunlap, G., Powell, D., \& Payne, C. J. (2014). An analysis of classroom-based and parentfocused social-emotional programs for young children. Infants \& Young Children, 27(1), 3-29. https://doi.org/10. 1097/IYC.0000000000000001

Belfield, C., Bowden, A. B., Klapp, A., Levin, H., Shand, R., \& Zander, S. (2015). The economic value of social and emotional learning. Journal of Benefit-Cost Analysis, 6(3), 508-544. https://doi.org/10.1017/bca.2015.55

Bierman, K. L., Domitrovich, C. E., Nix, R. L., Gest, S. D., Welsh, J. A., Greenberg, M. T., Blair, C., Nelson, K. E., \& Gill, S. (2008a). Promoting academic and social-emotional school readiness: The Head Start REDI program. Child Development, 79, 1802-1817. https://doi.org/10.1111/j.1467-8624.2008.01227.x

Bierman, K. L., Heinrichs, B. S., Welsh, J. A., Nix, R. L., \& Gest, S. D. (2017). Enriching preschool classrooms and home visits with evidence-based programming: Sustained benefits for low-income children. Journal of Child Psychology \& Psychiatry, 58(2), 129-137. https://doi.org/10.1111/jcpp.12618

Bierman, K. L., Nix, R. L., Greenberg, M. T., Blair, C., \& Domitrovich, C. E. (2008b). Executive functions and school readiness intervention: Impact, moderation, and mediation in the Head Start REDI Program. Development and Psychopathology, 20, 821-843. https://doi.org/10.1017/S0954579408000394

Bierman, K. L., Nix, R. L., Heinrichs, B. S., Domitrovich, C. E., Gest, S. D., Welsh, J. A., \& Gill, S. (2014). Effects of Head Start REDI on children's outcomes one year later in different kindergarten contexts. Child Development, 85, 140-159. https:// doi.org/10.1111/cdev.12117

Boykin, A. W. (1983). The academic performance of Afro-American children. In J. Spence (Ed.), Achievement and achievement motives (pp. 324-371). W. H. Freeman.

Brown, E. D., \& Sax, K. L. (2013). Arts enrichment and preschool emotions for low-income children. Early Childhood Research Quarterly, 28, 337-346. https://doi.org/10.1016/j.ecresq.2012.08.002

Burke, J. D., Loeber, R., Lahey, B. B., \& Rathouz, P. J. (2005). Developmental transitions among affective and behavioral disorders in adolescent boys. Journal of Child Psychology \& Psychiatry, 46(11), 1200-2120. https://doi.org/10.1111/j. $1469-7610.2005 .00422 . x$

Camilli, G., Vargas, S., Ryan, S., \& Barnett, W. S. (2010). Meta-analysis of the effects of early education interventions on cognitive and social development. Teachers College Record, 112(3), 579-620.

Collaborative for Social and Emotional Learning (CASEL). (2012). 2013 CASEL guide: Effective social and emotional learning programs_-Preschool and elementary school edition. http://www.casel.org/preschool-and-elementary-editi on-casel-guide

Committee on Developmental Outcomes and Assessments for Young Children. (2008). Early childhood assessment: Why, what, and how. The National Academies Press.

Conner, N. W., \& Fraser, M. W. (2011). Preschool social-emotional skills training: A controlled pilot test of the making choices and strong families program. Research of Social Work Practice, 21(6), 699-711. https://doi.org/10.1177/10497 31511408115

Consortium for Longitudinal Studies. (1983). As the twig is bent: Lasting effects of preschool programs. Lawrence Erlbaum.

Core SEL Competencies. (2019). https://casel.org/core-competencies/ 
D'Onise, K., McDermott, R. A., \& Lynch, J. W. (2014). Center-based preschool programs: Systematic review of child and adult health outcomes. In A. J. Reynolds, A. J. Rolnick, \& J. A. Temple (Eds.), Health and education in early childhood: Predictors, interventions, and policies (pp. 145-207). Cambridge University Press.

Darling-Churchill, K. E., \& Lippman, L. (2016). Early childhood social and emotional development: Advancing the field of measurement. Journal of Applied Developmental Psychology, 45, 1-7. https://doi.org/10.1016/j.appdev.2016.02.002

Denham, S. A., \& Burton, R. (1996). A social-emotional intervention for at-risk 4-year-olds. Journal of School Psychology, 34(3), 225-245. https://doi.org/10.1016/0022-4405(96)00013-1

Domitrovich, C. E., Cortes, R. C., \& Greenberg, M. T. (2007). Improving young children's social and emotional competence: A randomized trial of the preschool "PATHS" curriculum. The Journal of Primary Prevention, 28(2), 67-91. https://doi. org/10.1007/s10935-007-0081-0

Domitrovich, C. E., Greenberg, M. T., Kusche, C. A., \& Cortes, R. (2004). The PATHS preschool curriculum.

Duran, F. B., Hepburn, K. S., Kaufmann, R. K., \& Le, L. T. (n.d.). Research synthesis: Early childhood mental health consultation. The Center on the Social and Emotional Foundations for Learning.

Flook, L., Goldberg, S. B., Pinger, L., \& Davidson, R. J. (2015). Promoting prosocial behavior and self-regulatory skills in preschool children through a mindfulness-based kindness curriculum. Developmental Psychology, 51(1), 44-51. https:// doi.org/10.1037/a0038256

Garcia, J. L., Heckman, J. J., Leaf, D. E., \& Prados, M. J. (2016). The life-cycle benefits of an influential early childhood program. Human capital and economic opportunity working paper seriesThe University of Chicago.

Gormley, W. T., Phillips, D. A., Welti, K., Newmark, K., \& Adelstein, S. (2011). Social-emotional effects of early childhood education programs in Tulsa. Child Development, 82(6), 2095-2109. https://doi.org/10.1111/j.1467-8624.2011.01648.x

Greenberg, M. T., \& Kusche, C. A. (1993). Promoting social and emotional development in deaf children: The PATHS project. University of Washington Press.

Greenberg, M. T., Weissberg, R. P., O'Brien, M. U., Zins, J. E., Fredericks, L., Resnik, H., \& Elias, M. J. (2003). Enhancing schoolbased prevention and youth development through coordinated social and emotional learning. American Psychologist, 58, 466-474. https://doi.org/10.1037/0003-066X.58.6-7.466

Halle, T. G., \& Darling-Churchill, K. E. (2016). Review of measures of social and emotional development. Journal of Applied Developmental Psychology, 45, 8-18. https://doi.org/10.1016/j.appdev.2016.02.003

Hamre, B. K., Pianta, R. C., Mashburn, A. J., \& Downer, J.T. (2012). Promoting young children's social competence through the preschool PATHS curriculum and MyTeachingPartner professional development resources. Early Education and Development, 23(6), 809-832. https://doi.org/10.1080/10409289.2011.607360

Han, S. S., Catron, T., Weiss, B., \& Marciel, K. K. (2005). A teacher-consultation approach to social skills training for prekindergarten children: Treatment model and short-term outcome effects. Journal of Abnormal Child Psychology, 33(6), 681-693. https://doi.org/10.1007/s10802-005-7647-1

Han, H. S., \& Thomas, M. S. (2010). No child misunderstood: Enhancing early childhood teachers' multicultural responsiveness to the social competence of diverse children. Early Childhood Education Journal, 37(6), 469-476. https://doi.org/ 10.1007/s10643-009-0369-1

Hemmeter, M., Synder, P. A., Fox, L., \& Algina, J. (2016). Evaluating the implementation of the pyramid model for promoting social-emotional competence in early childhood classrooms. Topics in Early Childhood Special Education, 36(3), 133-146. https://doi.org/10.1177/0271121416653386

Hughes, C., \& Cline, T. (2015). An evaluation of the preschool PATHS curriculum on the development of preschool children. Educational Psychology in Practice, 31(1), 73-85.

Izard, C. E., King, K. A., Trentacosta, C. J., Morgan, J. K., Laurenceau, J. P., Krauthamer-Ewing, E. S., \& Finlon, K. J. (2008). Accelerating the development of emotion competence in Head Start children: Effects on adaptive and maladaptive behavior. Development and Psychopathology, 20(1), 369-397. https://doi.org/10.1017/S0954579408000175

Jensen, B., Jensen, P., \& Rasmussen, A. W. (2017). Does professional development of preschool teachers improve children's socio-emotional outcomes? Labour Economics, 45, 26-39. https://doi.org/10.1016/j.labeco.2016.11.004

Jones, D., Dodge, K. A., Foster, E. M., Nix, R., \& Conduct Problems Prevention Research Group. (2002). Early identification of children at risk for costly mental health service use. Prevention Science, 3(4), 247-256. https://doi.org/10.1023/A: 1020896607298

Jones, D. E., Greenberg, M., \& Crowley, M. (2015). Early social-emotional functioning and public health: The relationship between kindergarten social competence and future wellness. American Journal of Public Health, 105(11), 2283-2290. https://doi.org/10.2105/A.JPH.2015.302630

Joseph, G. E., \& Strain, P. S. (2003). Comprehensive evidence-based social-emotional curricula for young children: An analysis of efficacious adoption potential. Topics in Early Childhood Special Education, 23(2), 62-73. https://doi.org/10. $1177 / 02711214030230020201$

Karoly, L. A., \& Bigelow, J. H. (2005). The economics of investing in universal preschool education in California. Rand Corporation.

Kemple, K. M., Lee, I., \& Ellis, S. M. (2019). The impact of a primary prevention program on preschool children's social-emotional competence. Early Childhod Education Journal, 47, 641-652. https://doi.org/10.1007/s10643-019-00963-3

Keyes, C. L. M. (2002). The mental health continuum: From languishing to flourishing in life. Journal of Health and Social Research, 43, 207-222.

Klapp, A., Belfield, C., Bowden, B., Levin, H., Shand, R., \& Zander, S. (2017). A benefit-cost analysis of a long-term intervention on social and emotional learning in compulsory school. International Journal of Emotional Education, 9(1), 3-19.

Ladson-Billings, G. (1995). Toward a theory of culturally relevant pedagogy. American Educational Research Journal, 32(3), 465-491. https://doi.org/10.2307/1163320

Landry, S. H., Smith, K. E., Swank, P. R., \& Miller-Loncar, C. L. (2000). Early maternal and child influences on children's later independent cognitive and social functioning. Child Development, 71(2), 358-375. https://doi.org/10.1111/14678624.00150

Luo, L., Reichow, B., Snyder, P., Harrington, J., \& Polignano, J. (2020). Systematic review and meta-analysis of classroomwide social-emotional interventions for preschool children. Topics in Early Childhood Special Education. https://doi. org/10.1177/02171121420935579 
Lynch, K. B., Geller, S. R., \& Schmidt, M. G. (2004). Multi-year evaluation of the effectiveness of a resilience-based prevention program for young children. Journal of Primary Prevention, 24(3), 335-353. https://doi.org/10.1023/B:JOPP.00000 18052.12488.d1

Magnuson, K. A., Ruhm, C., \& Waldfogel, J. (2007). Does prekindergarten improve school preparation and performance? Economics of Education Review, 26(1), 33-51. https://doi.org/10.1016/j.econedurev.2005.09.008

McCabe, P. C., \& Altamura, M. (2011). Empirically valid strategies to improve social and emotional competence of preschool children. Psychology in the Schools, 48(5), 513-540. https://doi.org/10.1002/pits.20570

McCarthy, A., Lee, K., Itakura, S., \& Muir, D. W. (2006). Cultural display rules during eye gaze during thinking. Journal of Cross-Cultural Psychology, 37(6), 717-722. https://doi.org/10.1177/0022022106292079

McClelland, M. M., Tominey, S. L., Schmitt, S. A., \& Duncan, R. (2017). SEL interventions in early childhood. The Future of Children, 27(1), 33-47.

Merritt, E. G., Wanless, S. B., Rimm-Kaufman, S. E., Cameron, C., \& Peugh, J. L. (2012). The contribution of teachers' emotional support to children's social behaviors and self-regulatory skills in first grade. School Psychology Review, 41(2), 141-159. https://doi.org/10.1080/02796015.2012.12087517

Miller, T., \& Hendrie, D. (2008). Substance abuse prevention dollars and cents: A cost-benefit analysis. Center for Substance Abuse Prevention (CSAP), SAMHSA. DHHS Pub, (07-4298).

Moffitt, T. E., Arseneault, L., Belsky, D., Dickson, N., Hancox, R. J., Harrington, H., Houts, R., Poulton, R., Roberts, B. W., Ross, S., \& Sears, M. R. (2011). A gradient of childhood self-control predicts health, wealth, and public safety. Proceedings of the National Academy of Sciences, 108(7), 2693-2698. https://doi.org/10.1073/pnas.1010076108

Morris, P., Millenky, M., Raver, C., \& Jones, S. M. (2013). Does a preschool social and emotional learning intervention pay off for classroom instruction and children's behavior and academic skills? Evidence from the foundations of learning project. Early Education and Development, 24(7), 1020-1042. https://doi.org/10.1080/10409289.2013.825187

Murano, D., Sawyer, J. E., \& Lipnevich, A. A. (2020). A meta-analytic review of preschool social and emotional learning interventions. Review of Educational Research, 90(2), 227-263. https://doi.org/10.31092/0034654320914743

Muratori, P., Giuli, C., Bertacchi, I., Orsolini, L., Ruglioni, L., \& Lochman, J. E. (2017). Coping power for preschool-aged children: A pilot randomized control trial study. Early Intervention in Psychiatry, 11(6), 532-538. https://doi.org/10.1111/ eip.12346

National Institute for Early Education Research (NIEER). (2018). The state of preschool 2017. Rutgers.

Nelson, G., Westhues, A., \& MacLeod, J. (2003). A meta-analysis of longitudinal research on preschool prevention programs for children. Prevention and Treatment, 6, 1-34. https://doi.org/10.1037/1522-3736.6.1.631a

Newman, J., \& Dusenbury, L. (2015). Social and emotional learning (SEL): A framework for academic, social, and emotional success. In K. Bosworth (Ed.), Prevention science in school settings (pp. 287-306). Springer

Nix, R. L., Bierman, K. L., Heinrich, B. S., Gest, S. D., Welsh, J. A., \& Domitrovich, C. E. (2016). The randomized controlled trial of Head Start REDI: Sustained effects on developmental trajectories of social-emotional functioning. Journal of Consulting and Clinical Psychology, 84(4), 310-322. https://doi.org/10.1037/a0039937

Offord, D. R., Boyle, M. H., Racine, Y., Szatmari, P., Fleming, J. E., Sanford, M., \& Lipman, E. L. (1996). Integrating assessment data from multiple informants. Journal of the American Academy of Child and Adolescent Psychiatry, 35(8), 1078-1085. https://doi.org/10.1097/00004583-199608000-00019

Phillips, C. B. (1993). The movement of African American children through sociocultural contexts: A case of conflict resolution. In B. Mallory \& R. New (Eds.), Diversity \& developmentally appropriate practices (pp. 137-154). Teachers College Press.

Phillips, D. A., Lipsey, M. W., Dodge, K. A., Haskins, R., Bassok, D., Burchinal, M. R., Duncan, G. J., Dynarski, M., Magnuson, K. A., \& Weiland, C. (2017). Puzzling it out: The current state of scientific knowledge on pre-kindergarten effects. The Brookings Institution.

Pickens, J. (2009). Socio-emotional programme promotes positive behaviour in preschoolers. Child Care in Practice, 15(4), 261-278. https://doi.org/10.1080/13575270903149323

Poehlmann-Tynan, J., Vigna, A. B., Weymouth, L. A., Gerstein, E. D., Burnson, C., Zabransky, M., Lee, P., \& Zahn-Waxler, C. (2016). A pilot study of contemplative practices with economically disadvantaged preschoolers: Children's empathic and self-regulatory behaviors. Mindfulness, 7(1), 46-58. https://doi.org/10.1007/s12671-015-0426-3

Raver, C. C., \& Zigler, E. F. (1997). Social competence: An untapped dimension in evaluating Head Start's success. Early Childhood Research Quarterly, 12(4), 363-385. https://doi.org/10.1016/S0885-2006(97)90017-X

Reynolds, A. J., \& Ou, S. (2011). Paths of effects from preschool to adult well-being: A confirmatory analysis of the ChildParent Center Program. Child Development, 82(2), 555-582. https://doi.org/10.1111/j.1467-8624.2010.01562.x

Reynolds, A. J., Richardson, B. A., Hayakawa, M., Englund, M. M., \& Ou, S. (2016). Multi-site expansion of an early childhood intervention and school readiness. Pediatrics. https://doi.org/10.1542/peds.2015-4587

Reynolds, A. J., Temple, J. A., Ou, S., Arteaga, I. A., \& White, B. A. (2011). School-based early childhood education and age28 well-being: effects by timing, dosage, and subgroups. Science, 333(6040), 360-364. https://doi.org/10.1126/scien ce.1203618

Reynolds, A. J., Temple, J. A., Ou, S., Robertson, D. L., Mersky, J. P., Topitzes, J. W., \& Niles, M. D. (2007). Effects of a schoolbased, early childhood intervention on adult health and well-being: A 19-year follow-up of low-income families. Archives of Pediatrics and Adolescent Medicine, 161(8), 730-739. https://doi.org/10.1001/archpedi.161.8.730

Richardson, B. A., Reynolds, A. J., Temple, J. A., \& Smerillo, N. E. (2017). School readiness in the Midwest Child-Parent Center expansion: A propensity score analysis of year 1 impacts. Children and Youth Services Review, 79, 620-630. https://doi.org/10.1016/j.childyouth.2017.06.042

Riggs, N. R., Jahromi, L. B., Razza, R. P., Dillworth-Bart, J. E., \& Mueller, U. (2006). Executive function and the promotion of social-emotional competence. Journal of Applied Developmental Psychology, 27(4), 300-309. https://doi.org/10. 1016/j.appdev.2006.04.002

Rimm-Kaufman, S. E., Pianta, R. C., \& Cox, M. J. (2000). Teachers'judgments of problems in the transition to kindergarten. Psychology and Neuroscience, 15(2), 147-166. https://doi.org/10.1016/S0885-2006(00)00049-1 
Rutter, M., Kim-Cohen, J., \& Maughan, B. (2006). Continuities and discontinuities in psychopathology between childhood and adult life. Journal of Child Psychology and Psychiatry, 47(3-4), 276-295. https://doi.org/10.1111/j.1469-7610.2006. 01614.x

Shernoff, E. S., \& Kratochwill, T. R. (2007). Transporting an evidence-based classroom management program for preschoolers with disruptive behavior problems to a school: An analysis of implementation, outcomes, and contextual variables. School Psychology Quarterly, 22(3), 449-472. https://doi.org/10.1037/1045-3830.22.3.449

Stefan, C. A., \& Miclea, M. (2012). Classroom effects of a hybrid universal and indicated prevention program for preschool children: A comparative analysis based on social and emotional competence screening. Early Education and Development, 23(3), 393-426. https://doi.org/10.1080/10409289.2011.554756

Stefan, C. A., \& Miclea, M. (2013). Effects of a multifocused prevention program on preschool children's competencies and behavior problems. Psychology in the Schools, 50(4), 382-402. https://doi.org/10.1002/pits.21683

Totura, C. M., Green, A. E., Karver, M. S., \& Gesten, E. L. (2009). Multiple informants in the assessment of psychological, behavioral, and academic correlates of bullying and victimization in middle school. Journal of Adolescence, 32(2), 193-211. https://doi.org/10.1016/j.adolescence.2008.04.005

Upshur, C., Wenz-Gross, M., \& Reed, G. (2013). A pilot study of a primary prevention curriculum to address preschool behavior problems. Journal of Primary Prevention, 34, 309-327. https://doi.org/10.1007/s10935-013-0316-1

Washington State Institute for Public Policy. (2014). Early childhood education for low-income students: A review of the evidence and benefit-cost analysis. Washington State Institute for Public Policy.

Webb-Johnson, G. (2002). Are schools ready for Joshua? Dimensions of African-American culture among students identified as having behavioral/emotional disorders. International Journal of Qualitative Studies in Education, 15(6), 653-671. https://doi.org/10.1080/0951839022000014367

Webster-Stratton, C., \& Reid, M. J. (2004). Strengthening social and emotional competence in young children-The foundation for early school readiness and success - Incredible years classroom social skills and problem-solving curriculum. Infants \& Young Children, 17(2), 96-113. https://doi.org/10.1097/00001163-200404000-00002

Webster-Stratton, C. (1990). The teachers and children's videotape series: Dina Dinosaur's social skills and problem-solving curriculum. University of Washington Press.

Webster-Stratton, C. (1998). Preventing problems in Head Start children: Strengthening parent competencies. Journal of Consulting and Clinical Psychology, 66, 715-730. https://doi.org/10.1037/0022-006X.66.5.715

Webster-Stratton, C., Reid, J., \& Hammond, M. (2001). Preventing conduct problems, promoting social competence: A parent and teacher training partnership in Head Start. Journal of Clinical Child Psychology, 30, 283-302. https://doi. org/10.1207/S15374424JCCP3003_2

Webster-Stratton, C., Reid, M. J., \& Stoolmiller, M. (2008). Preventing conduct problems and improving school readiness: Evaluation of the incredible years teacher and child training program in high-risk schools. Journal of Child Psychology and Psychiatry, 49(5), 471-488. https://doi.org/10.1111/j.1469-7610.2007.01861.x

Weiland, C., \& Yoshikawa, H. (2013). Impacts of a prekindergarten program on children's mathematics, language, literacy, executive function, and emotional skills. Child Development, 84(6), 2112-2130. https://doi.org/10.1111/cdev.12099

Weissberg, R. P., Durlak, J. A., Domitrovich, C. E., \& Gullotta, T. P. (2016). Social and emotional learning: Past, present, and future. In J. A. Durlak, C. E. Domitrovich, R. P. Weissberg, \& T. P. Gullotta (Eds.), Handbook of social and emotional learning: Research and practice.Guildford Press.

West-Olatunji, C. A., Behar-Horenstein, L., Rant, J., \& Cohen-Phillips, L. N. (2008). Enhancing cultural competence among teachers of African American children using mediated lesson study. Journal of Negro Education, 77(1), 27-38.

Wille, N., Bettge, S., Wittchen, Ravens-Sieberer, U., \& BELLA Study Group. (2008). Risk and protective factors for children's and adolescents' mental health: Results of the BELLA Study. European Child \& Adolescent Psychiatry, 17, 133-147. https://doi.org/10.1007/s00787-008-1015-y

Williams, K. E., \& Berthelesen, D. (2019). Implementation of a rhythm and movement intervention to support self-regulation skills of preschool-aged children in disadvantaged communities. Psychology of Music, 47(6), 800-820. https:// doi.org/10.1177/0305735619861433

Williford, A. P., \& Shelton, T. L. (2008). Using mental health consultation to decrease disruptive behaviors in preschoolers: Adapting an empirically-supported intervention. Journal of Child Psychology and Psychiatry, 49(2), 191-200. https:// doi.org/10.1111/j.1469-7610.2007.01839.x

Wilson, K. R., Havighurst, S. S., \& Harley, A. E. (2012). Tuning in to kids: An effectiveness trial of a parenting program targeting emotion socialization of preschoolers. Journal of Family Psychology, 26(1), 56-65. https://doi.org/10.1037/a0026 480

Yates, T., Ostrosky, M. M., Cheatham, G. A., Fettig, A., Shaffer, L., \& Santos, R. M. (2008). Research synthesis on screening and assessing social-emotional competence. http://csefel.vanderbilt.edu/documents/rs_screening_assessment.pdf

Zigler, E., \& Trickett, P. K. (1978). IQ, social competence, and evaluation of early childhood intervention programs. American Psychologist, 33(9), 789-798. https://doi.org/10.1037/0003-066X.33.9.789

Zins, J. E., Bloodworth, M. R., Weissberg, R. P., \& Walberg, H. J. (2007). The scientific basis linking social and emotional learning to school success. Journal of Educational and Psychological Consultation, $17(2$ \& 3), 191-210. https://doi.org/10. 1080/10474410701413145

\section{Publisher's Note}

Springer Nature remains neutral with regard to jurisdictional claims in published maps and institutional affiliations. 